\title{
Epitaxial Graphene and Graphene-Based Devices Studied by Electrical Scanning Probe Microscopy
}

\section{Olga Kazakova ${ }^{1} *$, Vishal Panchal ${ }^{1,2}$ and Tim L. Burnett ${ }^{3}$}

1 National Physical Laboratory, Teddington TW11 0LW, UK; E-Mail: vishal.panchal@npl.co.uk

2 Royal Holloway University of London, Egham TW20 0EX, UK

3 Material Science Centre, University of Manchester, Manchester M1 7HS, UK;

E-Mail: timothy.burnett@manchester.ac.uk

* Author to whom correspondence should be addressed; E-Mail: olga.kazakova@ npl.co.uk; Tel.: +44-20-8943-6143; Fax.: +44-20-8614-0446.

Received: 10 December 2012; in revised form: 17 February 2013 / Accepted: 26 February 2013 / Published: 13 March 2013

\begin{abstract}
We present local electrical characterization of epitaxial graphene grown on both Si- and C-faces of $4 \mathrm{H}$-SiC using Electrostatic Force Microscopy and Kelvin Probe Force Microscopy in ambient conditions and at elevated temperatures. These techniques provide a straightforward identification of graphene domains with various thicknesses on the substrate where topographical determination is hindered by adsorbates and $\mathrm{SiC}$ terraces. We also use Electrostatic Force Spectroscopy which allows quantitative surface potential measurements with high spatial resolution. Using these techniques, we study evolution of a layer of atmospheric water as a function of temperature, which is accompanied by a significant change of the absolute surface potential difference. We show that the nanoscale wettability of the material is strongly dependent on the number of graphene layers, where hydrophobicity increases with graphene thickness. We also use micron-sized graphene Hall bars with gold electrodes to calibrate work function of the electrically conductive probe and precisely and quantitatively define the work functions for single- and double-layer graphene.
\end{abstract}

Keywords: epitaxial graphene; SiC; adsorbates; Kelvin Probe Force Microscopy (KPFM); Electrostatic Force Microscopy (EFM); surface potential; work function; wettability 


\section{Introduction}

Graphene, a single layer of graphite, is currently the subject of a massive research interest and an equally immense number of publications due to its novel physical properties and vast potential in technological applications: a likely successor of silicon in post-Moore's law devices, biochemical sensors, $\mathrm{THz}$ applications, etc. [1,2]. Graphene has also been found to be extremely valuable for metrological applications, for example, exceptionally accurate measurements of the quantum Hall resistance quantization were demonstrated recently [3]. In order to be economically viable and truly attractive for applications, large scale wafers of high quality graphene grown on insulating substrates are required. One of the most attractive routes is to grow graphene epitaxially from insulating $\mathrm{SiC}$ single crystals by solid-state graphitization of the substrate [4]. Besides wafer-scale graphene production (typically 2 " wafer [5,6], whereas 4" wafers are in a current research), the method provides a possibility to better control the electronic properties of graphene via charge transfer through interaction with the substrate.

However, during the high-temperature annealing process, the $\mathrm{SiC}$ substrate forms terraces with a typical height of 2-20 nm and eventually develops a complex surface morphology, which strongly depends on the growth conditions (temperature, gas atmosphere, pressure) as well as the initial miscut angle of the substrate. Most importantly, thermal decomposition of $\mathrm{SiC}$ is not a self-saturated process, which may result in the coexistence of graphene layers of various thicknesses. Thus, the SiC substrate significantly hinders straightforward identification and determination of the graphene layer thickness. For electronic applications in particular, it is crucial to define the number of graphene layers precisely as, for example, one and two layers of graphene (LG) are characterized by a completely different band structure and possibility to open the energy gap, defining the properties of devices. When morphology studies are performed in ambient conditions, the presence of water and various adsorbed species on the surface of graphene may further complicate the layer identification [7-9].

Topography measurements using Scanning Probe Microscopy (SPM) is a widely available and very versatile technique which has been extensively and successfully used for studies of the initial stages of graphitization on $\mathrm{SiC}$ [10-12] as well as for investigation of linear defects in epitaxial graphene, i.e., ridges, wrinkles/puckers, pleats, etc., which are generally the result of the compressive strain between graphene and SiC during cooling from the annealing process [13]. However, due to the complex morphology of the SiC substrate and inhomogeneity of the graphene growth, it is often very difficult, if not impossible, to precisely define the local thickness of the graphene studying the morphology alone. Confidence can be obtained by combination of topography height and tapping phase images [12,13], which in many cases can distinguish between different graphene domains (as discussed below). Nevertheless, many experimental studies still rely on the commonly available and simple height measurements as the main source of identification of the graphene thickness, which often leads to ambiguous and irreproducible results, especially in ambient conditions.

Besides the production of large-areas of graphene, forthcoming industrial needs require a large-scale contactless method for testing its electrical properties. Currently, only time-consuming, complicated and expensive electrical properties measurement methods of graphene are available, including patterning of devices and subsequent transport measurements. In this case, the obtained information is generalized over the whole device and not correlated with the exact morphology of 
graphene and presence of structural defects or local adsorbates. Electrical modes of SPM, for example Kelvin Probe Force Microscopy (KPFM) [14,15], Electrostatic Force Microscopy (EFM) [16], and Electrostatic Force Spectroscopy (EFS) [17] have recently been successfully used to identify the number of layers in epitaxial graphene. These techniques provide a contactless (and, therefore, simple, cheap and widely accessible) electrical mapping of epitaxial and exfoliated graphene allowing to extract crucial information about graphene thickness, distribution of the electrical potential and charge, work function, etc. on the nanoscale. While the EFM method allows mainly qualitative mapping of the surface potential [7,18-20], the KPFM technique provides quantitative values for the work function difference, $\Phi_{\mathrm{s}}=\Phi_{\text {probe }}-e V_{\mathrm{CPD}}$, where $\Phi_{\mathrm{s}}$ and $\Phi_{\text {probe }}$ are work functions of the surface and probe, respectively, and $V_{\mathrm{CPD}}$ is the contact potential difference directly measured by KPFM. Using KPFM method, both exfoliated [21] and epitaxial [22-24] graphene with different number of layers has been studied by various groups. In epitaxial graphene grown on $\mathrm{SiC}$, the method has proved to be useful for easy identification of graphene domains.

For numerous industrial applications (i.e., electrochemistry, electronic, environmental and chemical sensors, heterogeneous catalysis, supercapacitors, nanoparticle self-assembly, corrosion, lubricants, etc.) it is essential to know and control the state of the graphene surface. The presence of water is unavoidable when graphene is exposed to air for a prolonged period of time [25] and understanding its influence is crucial for understanding of both fundamental graphene properties and functionality of devices. Significant effort has been dedicated to both theoretical and experimental investigation of water on graphitic surfaces. In general, the hydrophobic nature of the graphene was commonly observed and revealed on the macroscale as a large contact angle, i.e., $\sim 93^{\circ}-120^{\circ}$, between a water droplet $(\sim 1 \mu \mathrm{L})$ and graphene surface, as measured by optical methods and X-ray reflectivity [26-28]. However, it has also been shown that few layer graphene on top of different substrates does not significantly change the wettability of such materials as gold, silicon and copper, owing to van der Waals forces dominating the surface-water interactions [29] and, as such, the few layer graphene appears hydrophilic in contradiction to bulk graphite. On the nanoscale, these interactions are even less understood.

Other mapping techniques have been successfully used for identification of graphene domains in past. Raman spectroscopy is a non-destructive technique, requiring virtually no sample preparation, is commonly used for determination of graphene quality and determination of the number of layers [30-32]. The technique directly measures the phonon dispersion bands related to the $A-B$ carbon atoms in the hexagonal lattice [33]. The most prominent features in the Raman spectra for graphene are the G-peak and the two dimensional (2D)-peak [32,34]. The G-peak $\left(\sim 1580 \mathrm{~cm}^{-1}\right)$ is a result of the doubly degenerate phonon modes which stems from the first-order scattering process. The 2D-peak $\left(\sim 2700 \mathrm{~cm}^{-1}\right)$ is a result of the double resonance electron-phonon scattering process which stems from the second-order scattering process [35]. Additionally, a defect peak D $\left(\sim 1350 \mathrm{~cm}^{-1}\right)$ is commonly observed for other than exfoliated flakes types of graphene [30,36,37]. The Raman spectra of epitaxial graphene on $\mathrm{SiC}$ produce additional peaks around $1300-1750 \mathrm{~cm}^{-1}$ due to the intrinsic phonon modes of the substrate, adding a further complication [32,38]. For precise analysis, one has to subtract the spectra for SiC. The Raman spectra features are highly specific to each material, acting as a unique chemical and structural fingerprint. This chemical sensitivity as a form of definitive material characterization is typically unmatched by electrical scanning probe microscopy techniques. A two 
dimensional (2D) Raman mapping is typically generated by performing spectroscopy at each point and extracting quantities such as peak position, relative intensities and the full width at half maximum (FWHM) values of the desired peaks. Contrast arises due to shifts in peak position and changes in the ratio of the intensities and FWHM [31,36], however, these properties can be affected by various extrinsic factors. The vibrational modes that are related to the $\mathrm{sp}^{2}$ and $\mathrm{sp}^{3}$ carbon bonds can be affected due to substrate induced stress and strain, thus affecting the Raman spectra [37]. The slow acquisition time of the Raman spectroscopy can significantly increase the mapping time, which can be optimized by increasing the power of the laser to increase the signal. However, this can lead to heating of the sample and potential damage [37]. The spatial resolution of Raman mapping is typically limited by the spot size of the laser to $\sim 1 \mu \mathrm{m}$, which is two orders of magnitude worse than electrical scanning probe microscopy techniques (10 s of nanometer). Often the layer non-uniformity of the epitaxial graphene on $\mathrm{SiC}$ is on the sub-micron scale, favoring electrical scanning probe microscopy techniques over Raman mapping.

Low energy electron microscopy (LEEM) and photoemission electron microscopy (PEEM) are other powerful techniques which are commonly used for local mapping of graphene properties in recent years. These techniques together with their spectroscopy counterparts, such as selected area or micro low energy electron diffraction ( $\mu$-LEED) and micro angle resolved photoelectron spectroscopy ( $\mu$-ARPES), were widely used for studies of graphene on metal, $\mathrm{SiC}$ and $\mathrm{Si} / \mathrm{SiO}_{2}$ substrates $[39,40]$. One of the main advantages of LEED/PEEM is a possibility to use it in real time for studies of such dynamic processes, as nucleation, growth and intercalation. These methods allow for the investigation of local properties, i.e., level of graphitization in spatially inhomogeneous graphene samples. For example, identification of single-layer and bilayer graphene films on a SiC substrate during the vacuum growth was performed by comparing the characteristic features in electron reflectivity spectra in LEEM to the $\pi$-band structure as revealed by ARPES [41]. In general, LEEM often serves as a very useful tool to accurately determine the local extent of graphene layers as well as the layer thickness. In particular, the role of the defective structure [42,43] and active treatment of the substrate [44] in formation of graphene layers were successfully studied. Another interesting approach is investigation of the effect of oxygen adsorption on the local structure and electronic properties of monolayer graphene. Monitoring of the oxidation process by LEEM and $\mu$-LEED demonstrated that the oxygen can saturate $\mathrm{Si}$ dangling bonds and break $\mathrm{Si}-\mathrm{C}$ bonds at the interface, leading to intercalation of the graphene layer [45]. Despite being extremely useful, these methods, however, require for expensive electronic microscopy equipment and essentially are vacuum techniques, which limits their application in product lines, where quick turn out of the samples and their simple and reliable characterization are crucial. Moreover, studies of adsorbed water and other atmospheric and environmental species are generally not possible in the vacuum environment.

Scanning Capacitance Microscopy (SCM) operates at microwave frequencies and has found widespread use in the analysis of dopant profiles in semiconductor materials [46,47]. SCM has been applied to the mapping of graphene in numerous instances and in slightly different ways. SCM has the ability to distinguish between different layer thicknesses of graphene and can also show further contrast within graphene domains. Different implementations have been used, for example, where the air-gap between the SPM tip and the graphene forms a metal-dielectric-metal series capacitor [48]. Alternatively, the tip-graphene forms the top electrode connected to the dielectric/semiconductor with 
the back gate (i.e., bottom electrode) being formed by heavily doped silicon substrate or additional metallic layer [49]. Lately it has been shown that using graphene in contact with the AFM tip as the top electrode provides a way of determining the mean free path in graphene as a bias is applied to the graphene for a finite period thus determining the area of the top electrode [50]. In each case the techniques involves significant analysis and de-convolution process is required to characterize the graphene individually as part of the whole measurement system. Currently there is still a relatively limited amount of work on the application of SCM to graphene and further systematic studies are needed to develop confidence in the technique when applied to graphene.

In this paper we review and summarize our results as well as results of others in the area of domain identification, studies of the surface potential distribution and precise determination of the work function in graphene domains of different thickness and type of doping as well as effects of environmental water and adsorbates on properties of epitaxial graphene. We focus our attention on local electrical studies of graphene epitaxially grown on $\mathrm{SiC}(0001)$ and $\mathrm{SiC}(000-1)$ as performed using a broad spectrum of electrical force microscopy techniques. Additionally, for lithographically prepared graphene devices we use local electrical mapping to complement standard transport measurements. All samples studied here are called "graphene" for simplicity, however we always specify the number of layers in each individual sample and describe thickness-dependent differences in their electronic and chemical properties.

The paper is organized as follows. In Part 2 we present our recent experimental results on (i) identification of epitaxial graphene domains and effect of adsorbates using quantified topography and electrical mapping; (ii) wettability of epitaxial graphene and influence of atmospheric water vapor and (iii) studies of graphene devices using KPFM and EFS techniques. Part 3 gives a brief overview of the used SPM techniques. Growth of graphene samples and fabrication of Hall bar devices are also outlined in Part 3. The summary of the results and the main conclusions are outlined in Part 4.

\section{Results and Discussion}

\subsection{Identification of Epitaxial Graphene Domains Using Quantified Topography Images}

Measuring the height of exfoliated graphene flakes deposited onto a substrate is often a convenient way of assessing the number of layers. However, even in this simple case care must be taken; for example, graphene deposited onto a gold substrate can appear depressed with respect to the substrate due to different electrostatic interaction with the sample based on the probe used and the materials being measured. Epitaxial graphene on $\mathrm{SiC}$ avoids this particular problem but there is a wide range of height values that can be expected between graphene domains, even those of the same thickness, as the substrate develops a complex morphology during growth [22,51]. The as grown film (sample \#1) studied in Sections 2.1 and 2.3 consisted of $\sim 57 / 40 / 3 \% 1$ LG/2 LG/3 LG, respectively, on $\mathrm{SiC}(0001)$.

\subsubsection{Models and Method}

We compare the expected height steps based on the model of Hass et al. [52] using the surface $\mathrm{X}$-ray reflectivity method and that predicted by Filleter et al. [22] and Emtsev et al. [10] using a conventional layer attenuation model (Table 1). Despite the intensive studies of epitaxial graphene on 
$\mathrm{SiC}$, the nature and properties of the interfacial layer (IFL) are not very well understood. However, it is clear that this layer plays a defining role in the morphological and electronic properties of epitaxial graphene. At present, the consensus is that IFL is a $(6 \sqrt{3} \times 6 \sqrt{3}) \mathrm{R} 30^{\circ}$ reconstruction of $\mathrm{SiC}(0001)$. The layer is carbon-rich and covalently bonded to the substrate. The incomplete understanding of the IFL inevitably causes different interpretation of its thickness. For example, values of $t_{\mathrm{IFL}} \approx 0.232 \mathrm{~nm}$ [52], $t_{\mathrm{IFL}} \approx 0.250 \mathrm{~nm}[22]$ and $0.240 \pm 0.030 \mathrm{~nm}$ [10] have been predicted. Recently, it was argued that the thickness of the IFL is not constant but dependent on the thickness of the graphene layer on top, giving rise to a very broad range of values $t_{\mathrm{IFL}} \approx 0.150-0.900 \mathrm{~nm}[53]$.

Table 1. The range of heights in nanometers expected between 1 LG (layer of grapheme) and 2 LG in epitaxial graphene on SiC using the two models shown. The minus sign denotes when the $1 \mathrm{LG}$ is higher with respect to the $2 \mathrm{LG}$.

\begin{tabular}{ccc}
\hline Graphene layers & Model used & Step height $(\mathbf{n m})$ \\
\hline \multirow{2}{*}{$1 \mathrm{LG}-2 \mathrm{LG}$} & Filleter et al. $[22]$ & $-0.915,-0.665,-0.415,-0.165,+0.085,+0.335,+0.585$, \\
& & $+0.835,+1.085$ \\
& & $-0.925,-0.673,-0.421,-0.169,+0.083,+0.335,+0.587$, \\
1 LG-2 LG & Hass et al. $[52]$ & $+0.839,+1.092$ \\
& & $-1.065,-0.815,-0.565,-0.315,-0.065,+0.185,+0.435$, \\
1 LG + 0.400 nm-2 LG & Burnett et al. [54] & $+0.685,+0.935$ \\
\hline
\end{tabular}

In order to compare the height measurements, we first had to confirm the most reliable experimental method of measuring vertical distances on a nanometre scale. Three different methods were considered for determination of the vertical heights using AFM topography measurements, namely: individual line profiles, averaged line profiles based on recording of 51 individual parallel lines, and histogram plots. We have previously confirmed that the histogram method of measuring the small height steps between epitaxial graphene domains is the most reliable [54]. To use the histogram method it is crucial to adjust the tilt of the image to produce flattened steps with constant values across the graphene domains, whilst ensuring that, with an exception of tilting adjustment, no flattening procedures were applied to the images [54]. The appropriate level of tilting is achieved when the histogram can be accurately fitted by a Gaussian distribution. The histogram method is especially versatile as it is possible to ignore spurious contributions to the measured height such as depressions and surface adsorbates, which are not easy to avoid with line trace methods. The histogram method was then used to explore the consistency of the height measurements between domains on epitaxial graphene samples. It is also important to ensure that the topography measurements are made with all regions imaged within the repulsive regime during the mechanical oscillation utilized in tapping mode [55] (see Section 3.1). Epitaxial graphene is relatively straightforward in this respect as we have crystallographic steps of known height on the substrate that are covered with the same material which can be used as a reference point. 


\subsubsection{Experimental Results}

Initially, we consider the height of $\mathrm{SiC}$ terraces and the case when graphene domain homogeneously covers a few SiC terraces. The step height across terraces was always measured as a multiple of $0.250 \mathrm{~nm} \pm$ a conservative value of $0.050 \mathrm{~nm}$ (in recognition of the noise floor of the AFM, although the experimental values were measured within a few picometers). For example, a measured terrace height of $0.995 \mathrm{~nm}$ was compared to the model value of $1.000 \mathrm{~nm}$ in almost perfect agreement to the expected height of four layers of $\mathrm{SiC}$ as predicted for a single $\mathrm{SiC}$ layer height of $0.250 \mathrm{~nm}$ [22]. This was found to be consistent, independent of the graphene layer thickness on top of the terraces (up to the $3 \mathrm{LG}$ ), confirming the conformity of the graphene to the underlying $\mathrm{SiC}$ and uniformity of the graphene domains and once a suitable tip and measurement parameters had been established the repeatability was typically well within $\pm 0.020 \mathrm{~nm}$ giving us a large degree of confidence in our height measurements.

However, the height variations between different graphene domains have little relation to the values expected from theory as shown in Table 1. By assuming an adsorbed layer with a thickness of $0.400 \mathrm{~nm}$ adhered only to the $1 \mathrm{LG}$, we found that the heights measured between $1 \mathrm{LG}$ and $2 \mathrm{LG}$ come to an extremely close agreement with the theory introduced in Section 2.1.1. For a large range of measurements on different $1 \mathrm{LG}$ and $2 \mathrm{LG}$ samples on $\mathrm{SiC}(0001)$, the $0.400 \mathrm{~nm}$ adjustment proved to be an accurate and consistent correction, matching the theory with the measurements [54] typically within $0.050 \mathrm{~nm}$. Larger discrepancies were sometimes found but the corrected value always provided a significantly better match than the uncorrected theoretical values. This correction allows us to use current theories on the expected height steps and match them to the experimental results. Within this margin of error both theories presented in Table 1 match the measurements with close agreement after correction. We discuss the nature of this adsorbed layer in Sections 2.2 and 2.3, especially in regard to Figure 3. We show that the thickness of the adsorbed layers is extremely consistent for our samples which have been prepared in the same way on nominally the same starting substrates. Figure 1 shows topography and corresponding EFM phase images (Section 3.2) for two different regions, where the height is measured using the histogram method. The histogram of the highlighted area in Figure 1a is depicted in Figure 1c, where the 2 LG is lower with respect to the $1 \mathrm{LG}$ and a height of $0.599 \mathrm{~nm}$ is measured between them. Referring to Table 1 , this height relates to $-0.165 \mathrm{~nm}$ (the minus sign denotes the $2 \mathrm{LG}$ is lower than $1 \mathrm{LG})$. Once we add the $0.400 \mathrm{~nm}$ related to an adsorbed surface layer, the value is $-0.565 \mathrm{~nm}$. This is in excellent agreement with our measured value. For Figure 1d the height measured between the $1 \mathrm{LG}$ and $2 \mathrm{LG}$ is $0.753 \mathrm{~nm}$, in this case the $1 \mathrm{LG}$ domain is lower than 2 LG. Referring again to Table 1, this value is in a good agreement with the height of $0.685 \mathrm{~nm}$ which we get when $0.400 \mathrm{~nm}$ is subtracted from a value of $1.085 \mathrm{~nm}$. Although this agreement is not perfect, it is by far the closest solution and shows that the method is reliable. Further examples can be found in [54], altogether showing the robustness of the histogram technique and the need to account for an adsorbed surface layer, which in this case can be assigned a thickness of $\sim 0.400 \mathrm{~nm}$, but might be different for different samples and preparation routes. 
Figure 1. (a) Two different regions of sample \#1; (a) and (d) Topography, the boxes show location of the histogram analysis; (b) and (e) Electrostatic Force Microscopy (EFM) phase images of the areas in (a) and (d), obtained with $V_{\text {probe }}=+2 \mathrm{~V}(\mathbf{b})$ and $-2 \mathrm{~V}(\mathbf{e})$. as the $V_{\text {probe }}$ is different for the two images, the $2 \mathrm{LG}$ is darkest in (b); whereas the $1 \mathrm{LG}$ is darkest in (e), which also contains some very bright 3 LG domains; (c) and (f) Height histograms of the highlighted areas in (a) and (d).
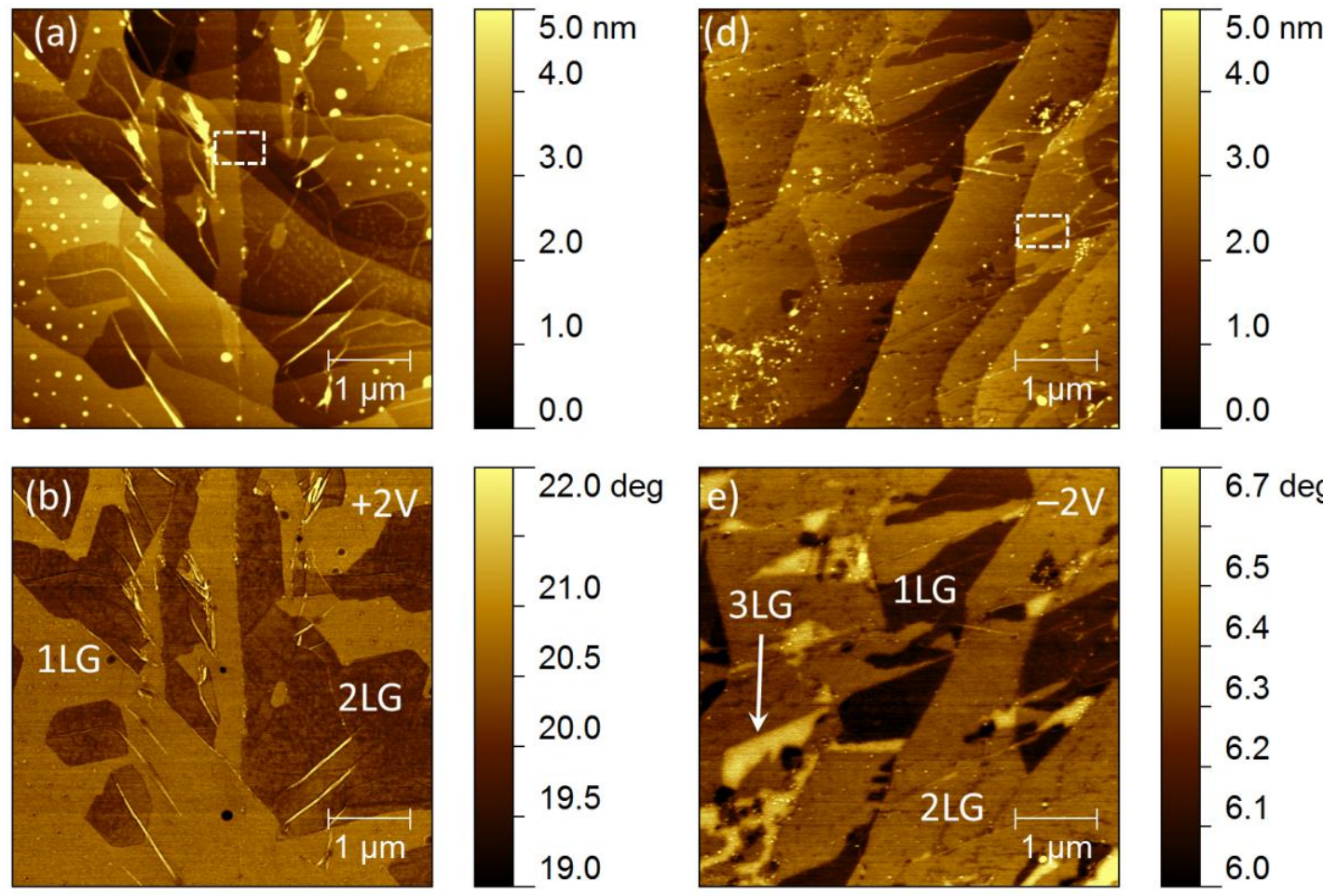

(c)

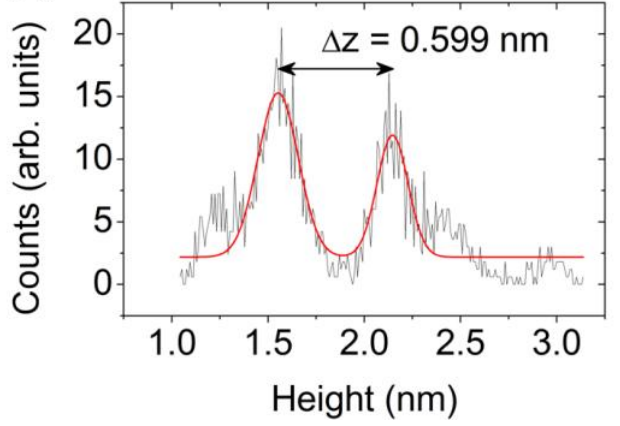

(f)

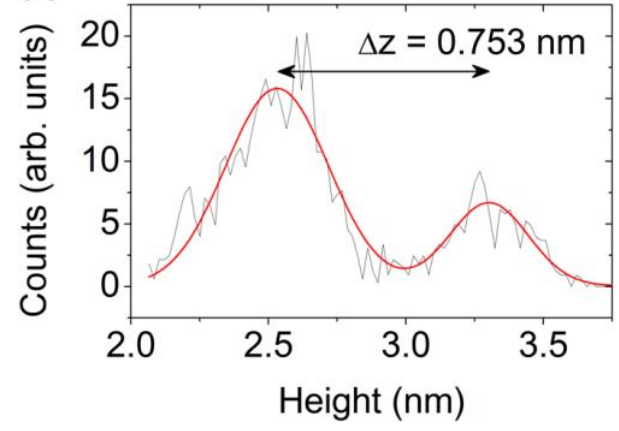

\subsection{Mapping of Local Electrical Properties in Epitaxial Graphene}

\subsubsection{Experimental Results}

As described above, the identification of different graphene domains using quantitative AFM is generally possible with careful analysis of the step heights between domains and in our case also including a correction for an adsorbed surface layer. However, the use of electrical modes of SPM can provide a much easier method to delineate different graphene domains through imaging alone (Figure 1b,e). Variation in electrical properties for graphene domains with different layer thicknesses 
offers the means to distinguish between layers using EFM and KPFM. The images shown in Figure 2 are a small section of device \#4, which will be discussed in more detail in Section 2.4.3.

Figure 2. Series of images from the same region showing 1 LG with a small 2 LG island: (a) topography; (b) tapping phase showing 1 LG and 2 LG regions; (c) EFM phase at $V_{\text {probe }}=+2 \mathrm{~V}$; (d) Electrostatic Force Microscopy (EFM) phase at $V_{\text {probe }}=-2 \mathrm{~V}$; (e) Electrostatic force spectroscopy (EFS) curves for the 1 LG and 2 LG; (f) Kelvin Probe Force Microscopy (KPFM) surface potential map.
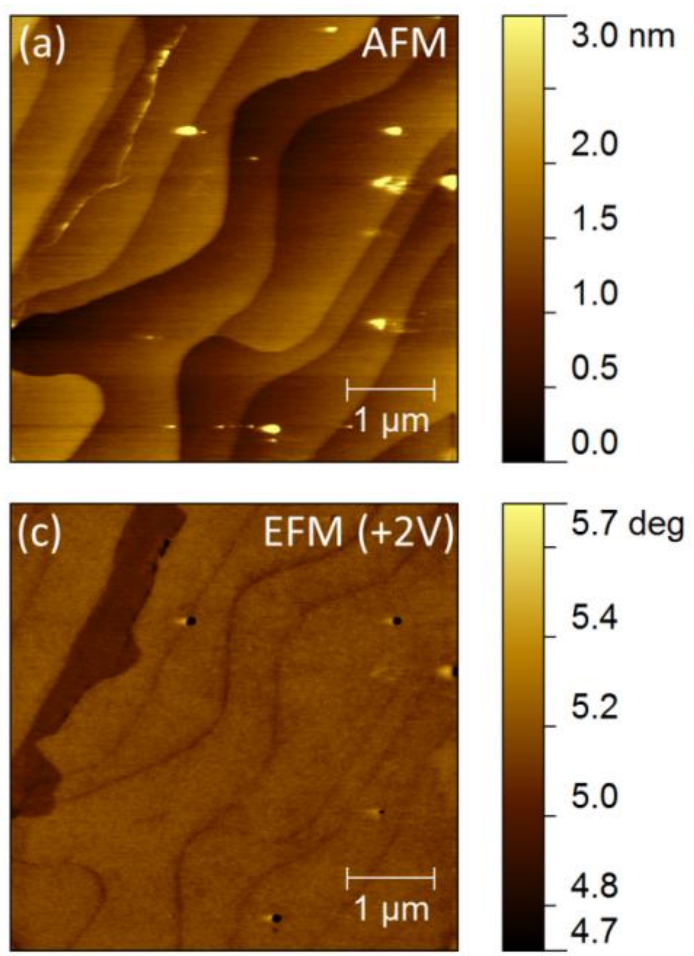

(e)

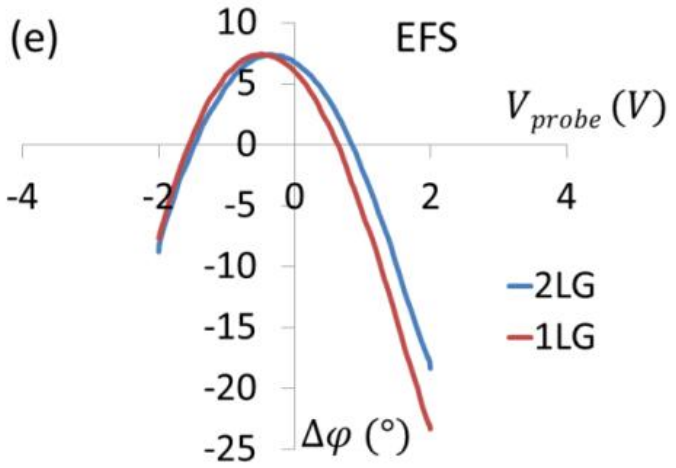

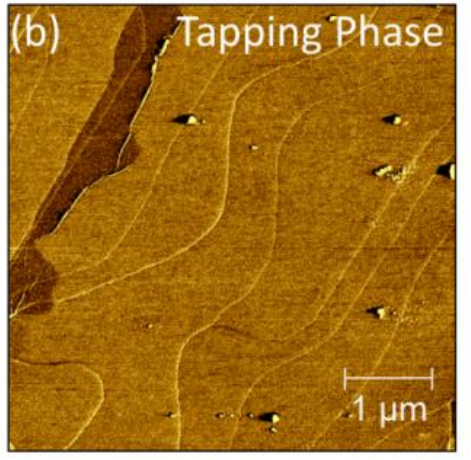
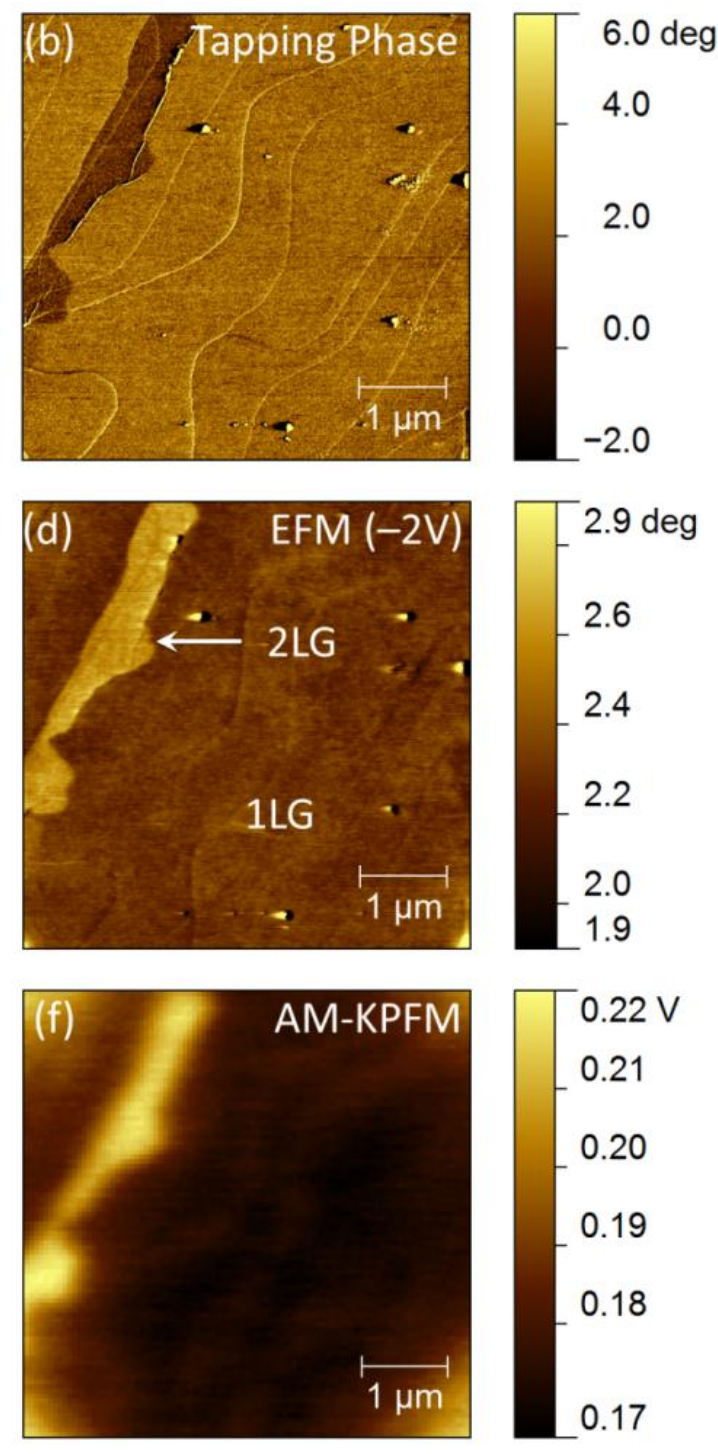

While the topography image (Figure 2a) is strongly dominated by SiC terraces, hindering information about the graphene thickness, the tapping phase image (Figure 2b) allows identification the 2 LG domain. Figure 2c,d show two images recorded using EFM with different probe biases ( $V_{\text {probe }}$ ). The contrast between the $1 \mathrm{LG}$ and $2 \mathrm{LG}$ is flipped when the bias is changed from +2 to $-2 \mathrm{~V}$ (additionally the phase difference increases from $0.63^{\circ}$ to $1^{\circ}$ ). This ability to manipulate the contrast levels is very valuable for clear identification of different domains, as there is always the possibility to validate a result by recording multiple images at different biases. However, a more succinct way of identifying the optimum contrast between graphene domains can be achieved utilizing EFS 
(Section 3.3). For the images in Figure 2c,d, multiple EFS measurements were made and averaged to produce the graph shown in Figure 2e. Each of the graphene domains shows a distinct parabola, plotting EFM phase $(\varphi)$ as a function of the $V_{\text {probe }}$. At $V_{\text {probe }}=-2 \mathrm{~V}$, the EFS curve for the 2 LG has a larger $\Delta \varphi$ than the $1 \mathrm{LG}$, which translates to the $2 \mathrm{LG}$ appearing the brightest part of the image in Figure $2 \mathrm{~d}$. At $V_{\text {probe }}=+2 \mathrm{~V}$, the $2 \mathrm{LG}$ has a lower phase than the $1 \mathrm{LG}$ and is, therefore, darker in the EFM phase images. Thus, it is possible to use EFS to find optimal imaging parameters to maximize the contrast in the images. We have also demonstrated a high reliability of the EFS technique, as results of averaging of a large number of individual curves typically show a very small standard deviation, i.e., within $\sim 3 \%$ of the mean value as was measured for various types of graphene samples and different experimental conditions for a statistical range of 50-46,000 EFS curves. Additionally, the quantitative mapping of the same area has been obtained using AM-KPFM technique (Figure 2f), showing the surface potential difference between $1 \mathrm{LG}$ and $2 \mathrm{LG}$, being $\Delta V_{\mathrm{CPD}} \sim 40 \mathrm{mV}$.

\subsubsection{Discussion}

\subsubsection{Effect of the Substrate on the Electronic Properties of Epitaxial Graphene}

The possibility of unambiguously identifying the graphene layer thickness and, furthermore, inferring fundamental properties of the graphene under ambient conditions is limited. This limitation is introduced primarily by the sensitivity of graphene to its immediate environment which induces a large degree of changeability in its physical properties. It is commonly accepted that in the case of graphene grown on the $\mathrm{SiC}(0001)$, the substrate and the IFL are a strong source of electrons, leading to intrinsic n-doping of epitaxial graphene. 2 LG, which is less strongly influenced by the substrate, has a lower level of $n$-doping with this trend continuing with increasing layer thickness. The intrinsic doping level originating from the substrate has been explored for exfoliated graphene on $\mathrm{SiO}_{2}$ [21]. We expect essentially the same trend for epitaxial graphene, bearing in mind a different type of dopants and presence of the IFL.

\subsubsection{Effects of Structural Defects and Strain}

The work function measured with KPFM and EFS is directly related to the carrier concentration of the graphene and, therefore, the doping level is fundamental to the measured surface potential. There is a large range of $V_{\mathrm{CPD}}$ values reported in the literature (Table 4, Section 2.4.3) as can be expected based on intrinsic doping alone. Even an explicit and detailed knowledge of the substrate-related doping does not necessarily guarantee a reasonable agreement with the experimentally measured carrier concentration in the graphene. The level of doping is also dictated by the geometrical relationship of the graphene to the substrate. In exfoliated graphene it has been shown that the closer the graphene is to the substrate the greater the charge interaction between them, with annealing improving the graphene-substrate conformity. An undetermined roughness is not expected in the case of epitaxial graphene, as the IFL and graphene grow directly on the atomically flat SiC surface. Nevertheless, domains of different layer thickness have an 'intrinsic' roughness [56], which is likely to influence the graphene-IFL-substrate interaction. Substrate steps also influence the charge interaction, where the terrace width and height are both dependent on the exact growth conditions [57] and the miscut of the 
original $\mathrm{SiC}$ crystal [58]. It was shown that when the graphene passes over a terrace edge, a detachment of the graphene from the substrate is possible leading to an abrupt change in local doping [59], which can be seen with KPFM and EFM as a change in the potential (Figure 2). However, this effect should be distinguished from the possibility of narrow MLG bands nucleating at the terrace edge [60]. Other structural features and defects that are found on epitaxial graphene (e.g., puckers formed due to the thermal coefficient of expansion mismatch between the graphene and the substrate [61]), also have an appreciable effect on the electrical properties. Such defects have been shown to strongly influence the device properties depending on whether the alignment of the devices crossed the wrinkles perpendicularly or were parallel to them [62].

The influence of strain, which occurs during cooling of epitaxial graphene (also during the mechanical deposition of exfoliated graphene [51]), has also been highlighted as intimately related to the charge transfer process from the substrate [63], which is supported by the fact that both the geometry of the graphene and proximity to the substrate influence the transfer of charge.

\subsubsection{Influence of Adsorbates}

There are a multitude of molecules that may become adsorbed to the graphene surface even based purely on exposure to the ambient laboratory environment (typically hydrocarbon-based unidentified molecules [64]). In our own work we have made preliminary studies using Secondary Ions Mass Spectroscopy (SIMS) which shows the presence of polydimethylsiloxane (PDMS) as a surface contaminant with traces of erucamide and dimyristoylphophatidylcholine [33]. These species are likely to be responsible for the formation of zero dimensional adsorbates (Figure 1a) on the sample surface based on the previous studies showing the formation of siloxane films on carbon surfaces $[65,66]$. These compounds are likely to originate from the environment of plastic storage containers, gloves, and numerous other applications products where PDMS is used in production as a mold release agent. No water was observed due to the fact that the SIMS measurements were carried out in high vacuum. Effects from atmospheric water and resist residues from lithography processes are specifically discussed in Sections 2.3 and 2.4, respectively.

All of these factors lead to a daunting array of contributions to the exact level of intrinsic doping expected in the graphene and measured using EFS and KPFM. Assurance can be attained through complementing the measurements with Raman Spectroscopy, LEEM, ARPES or another technique to give a baseline for the subsequent SPM measurements to confirm 1 LG coverage. However, we are successfully working on creating a high level of confidence with SPM in ambient conditions as a technique which can be relied upon to a high level of certainty.

\subsection{Wettability of Epitaxial Graphene and Effect of Atmospheric Water}

Exposure to atmospheric environment may significantly alter the electronic properties of graphene and functionality of graphene-based devices due to an additional extrinsic doping [67-69], where such common species as water vapor, oxygen, nitrogen and carbon oxides and hydrocarbons act as p-dopants, see e.g., [70-77]. However, high reactivity and instability of many of these dopants make their practical application rather limited [70]. The effect of variable humidity on properties of graphene has received limited attention. SPM techniques have been widely used in the past for studies of thin 
water layers on various substrates in ambient conditions. While the AFM probe may strongly perturb the nanoscale liquid film, the use of non-contact modes was shown to significantly reduce the friction and dragging effects. It was generally found that initially an ordered, ice-like layer of structured water, characterized by the lack of free $\mathrm{OH}$ groups, can be formed even at ambient temperature and humidity on both hydrophilic and even hydrophobic substrates [78]. The AFM technique is very useful for successful visualization and investigation of such structures. Here, we use a variety of SPM modes to study the process of absorption and desorption of water on graphene and relevant modification of graphene surface potential. While SPM techniques cannot provide a direct chemical proof that the adsorbed species are indeed related to atmospheric water, a set of experiments outlined below gives us a high level of confidence in their nature. It should also be noted that a direct chemical analyses of the adsorbed species is extremely problematic on nanoscale and, in particular, in ambient conditions.

\subsubsection{Case of Epitaxial Graphene on Si-Face SiC}

First, we consider the influence of atmospheric water on properties of epitaxial graphene grown on the $\mathrm{Si}$-face of $\mathrm{SiC}$ in the temperature range $20-120{ }^{\circ} \mathrm{C}$ and at $\sim 40 \%$ relative humidity. In this section sample \#1 is studied. The tapping phase images demonstrate that 1 LG has a uniform and homogeneous surface. However, a substructure associated with the presence of adsorbed water can clearly be seen within 2 LG and 3 LG domains at room temperature, which in the case of the 3 LG domain form a well-organized pattern (Figure 3a, RT1 denotes initial room temperature measurements). Individual droplets are $\sim 50-80 \mathrm{~nm}$ in width and $\sim 0.4-0.8 \mathrm{~nm}$ in height, though the exact measurements are hindered by the small size and closely packed position of the droplets. As the temperature increases, the surface of $2 \mathrm{LG}$ and $3 \mathrm{LG}$ changes considerably. At $50{ }^{\circ} \mathrm{C}$, the substructure within the $3 \mathrm{LG}$ domain is already modified and can be characterized by fewer and larger features. At $80{ }^{\circ} \mathrm{C}$, the substructure disappears completely and both $2 \mathrm{LG}$ and $3 \mathrm{LG}$ domains become almost entirely uniform (Figure 3b). As the temperature returns back to $20{ }^{\circ} \mathrm{C}$, the substructure reappears on both $2 \mathrm{LG}$ and $3 \mathrm{LG}$ (Figure 3c, RT2 denotes the final room temperature measurement). Moreover, a very similar but not entirely identical pattern consisting of parallel lines can be clearly seen on 3 LG, demonstrating complete reversibility of the process and confirming that the patterns are not a measurement artifact. The tapping phase contrast for the $1 \mathrm{LG}$ domain is generally temperature independent, with a standard deviation from the mean value being $\Delta \varphi \sim 0.25^{\circ}$ in the whole temperature range. However, for 2-3 LG this parameter changes significantly with temperature, being in the range $0.32^{\circ}-0.66^{\circ}$. As the tapping phase technique is sensitive to the energy dissipation on the surface, this analysis implies the presence of an additional layer of a different material on top of 2-3 LG domains and its modification with temperature. Comparison of topography line profiles taken at $\mathrm{RT} 1,80{ }^{\circ} \mathrm{C}$ and RT2 across 1 LG and 2 LG domains is shown in Figure 3e. While the surface roughness of 1 LG is virtually temperature independent (in agreement with tapping phase results), 2 LG shows significantly higher features at RT1 and RT2 (with a height of $\sim 0.400 \mathrm{~nm}$ ), which is consistent with thickness of the monolayer of water. At $80{ }^{\circ} \mathrm{C}$ the features on the $2 \mathrm{LG}$ domain become significantly lower in height, indicating disappearance of the water. Upon the following cooling down to RT2, the initial roughness of the 2 LG is restored as water is re-absorbed [9]. 
Figure 3. Tapping phase images $1 \mathrm{LG}, 2 \mathrm{LG}$ and $3 \mathrm{LG}$ domains on $\mathrm{SiC}(0001)$ obtained at (a) RT1; (b) $80{ }^{\circ} \mathrm{C}$ and (c) RT2, showing process of desorption and re-adsorption of atmospheric water. The contour of $3 \mathrm{LG}$ is highlighted using a white line in (a) for clarity; (d) Topography image at RT1, water appears as lighter contrast on 2-3 LG; (e) Topography profiles along the line shown (d) obtained at RT1, $80^{\circ} \mathrm{C}$ and RT2. Profiles are vertically offset for clarity. The vertical arrow indicates the position of the SiC terrace of $\sim 1 \mathrm{~nm}$ height dividing the $1 \mathrm{LG}$ and 2 LG domains. The vertical dashed lines show an approximate border between 2 LG and 3 LG domains. The horizontal dashed lines define the spatial resolution of the method, corresponding to $2 \sigma$-value of the Gaussian distribution.
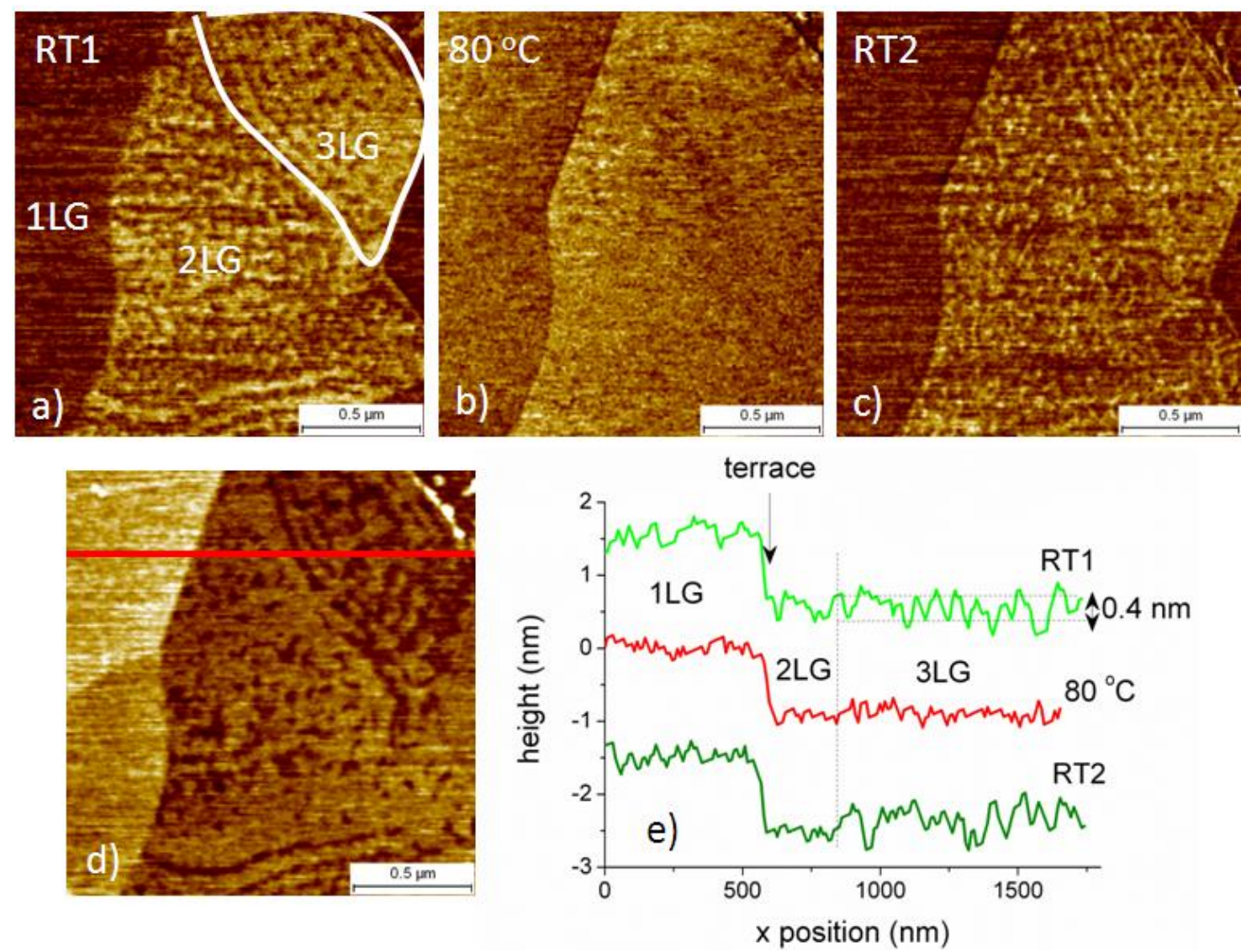

As the atmospheric water molecules adsorb on the sample surface $[67,70,79]$, they affect the overall surface potential, $V_{\mathrm{CPD}}$, of the graphene. In the following set of experiments, we investigate modification of the surface potential of graphene as a result of the temperature change and corresponding transformation of the water layer. The area shown in Figure 4a was monitored through the series of temperatures, i.e., $20 \rightarrow 120 \rightarrow 20^{\circ} \mathrm{C}$ using a 2-pass AM-KPFM mode (see Section 3.4). Linear profiles of the potential images obtained at 20 and $120^{\circ} \mathrm{C}$ are shown in Figure $4 \mathrm{~b}$. The Figure demonstrates that, while $V_{\mathrm{CPD}}$ of $1 \mathrm{LG}$ is generally not affected by the temperature change, the surface potential of $2 \mathrm{LG}$ and $3 \mathrm{LG}$ significantly increases as the temperature rises. The summary of $\Delta V_{\mathrm{CPD}}$ measurements is presented in Figure 4c. For the 1 LG-2 LG area, the $\Delta V_{\text {CPD }}$ steadily increases with temperature, reaching $\sim 50 \mathrm{mV}$ at $120{ }^{\circ} \mathrm{C}$. Once the sample was cooled back down to room temperature, $\Delta V_{\mathrm{CPD}}$ also returned to its initial value. A different behavior was observed for 2-3 LG 
area: First $\Delta V_{\mathrm{CPD}}$ sharply increases at $50{ }^{\circ} \mathrm{C}$ and as the temperature increases further, $\Delta V_{\mathrm{CPD}}$ drops back to the initial value.

Figure 4. (a) Room temperature AM-KPFM image of graphene on $\mathrm{SiC}(0001)$ showing 1 LG, 2 LG and 3 LG domains; (b) Linear profiles of the surface potential difference at room temperature (RT), 80 and $120^{\circ} \mathrm{C}$ along the line shown in (a). Vertical dotted lines show the approximate boundaries of domains; (c) Temperature dependence of the change of the surface potential difference obtained using histogram analyses of subsequent surface potential images for 1 LG-2 LG and 2 LG-3 LG domains. The arrows show directions of the temperature change. The solid lines are guides for the eye.
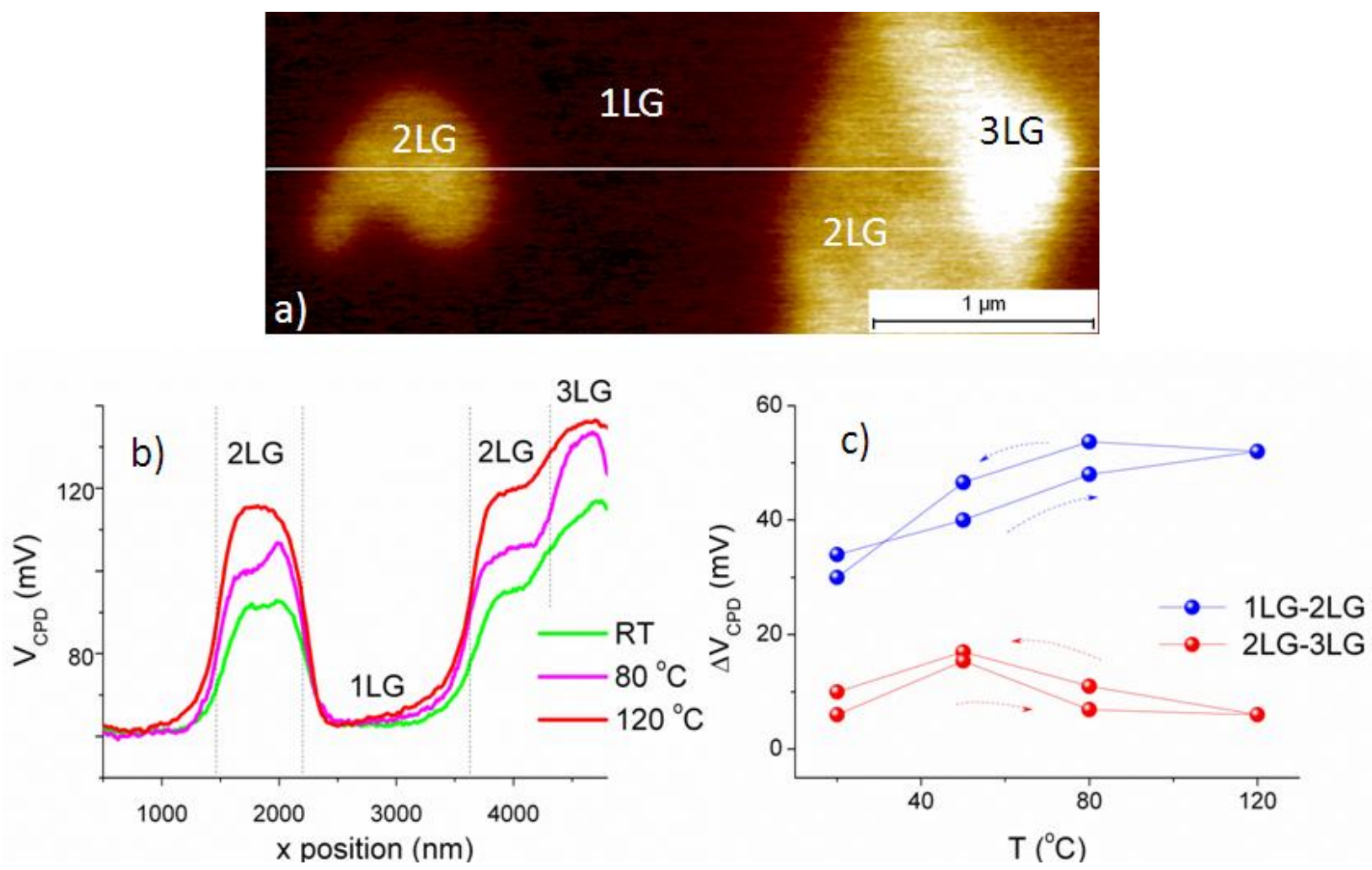

\subsubsection{Case of Epitaxial Graphene on C-Face $\mathrm{SiC}$}

A similar experiment on the influence of atmospheric water was performed on epitaxial graphene grown on the C-face of SiC (sample \#2). We consider a $5 \times 5 \mu \mathrm{m}^{2}$ area consisting of few ( 3-4 LG) and many ( 9-10 LG) layers graphene domains (FLG and MLG, respectively) and follow the transformation of morphology and surface potential difference as a function of temperature (Figure 5). The number of layers was defined by deliberately scribing off the layer of graphene from the substrate using a contact-mode AFM (CM-AFM) with a high force deliberately applied to the stiff cantilever and measuring the resulting step by applying the histogram method [54]. 
Figure 5. Topography images of graphene on $\mathrm{SiC}(000-1)$ showing FLG-MLG domains at (a) RT and (b) $120{ }^{\circ} \mathrm{C}$. Left column - the scan size is $\sim 5 \mu \mathrm{m}$; right column - magnified area within the frame (highlighted in the left column) showing transformation of water with temperature. Areas of "liquid" and "solid" water are marked; (c) Line profiles of the topography at RT and $120{ }^{\circ} \mathrm{C}$ along the lines shown in (a) and (b).

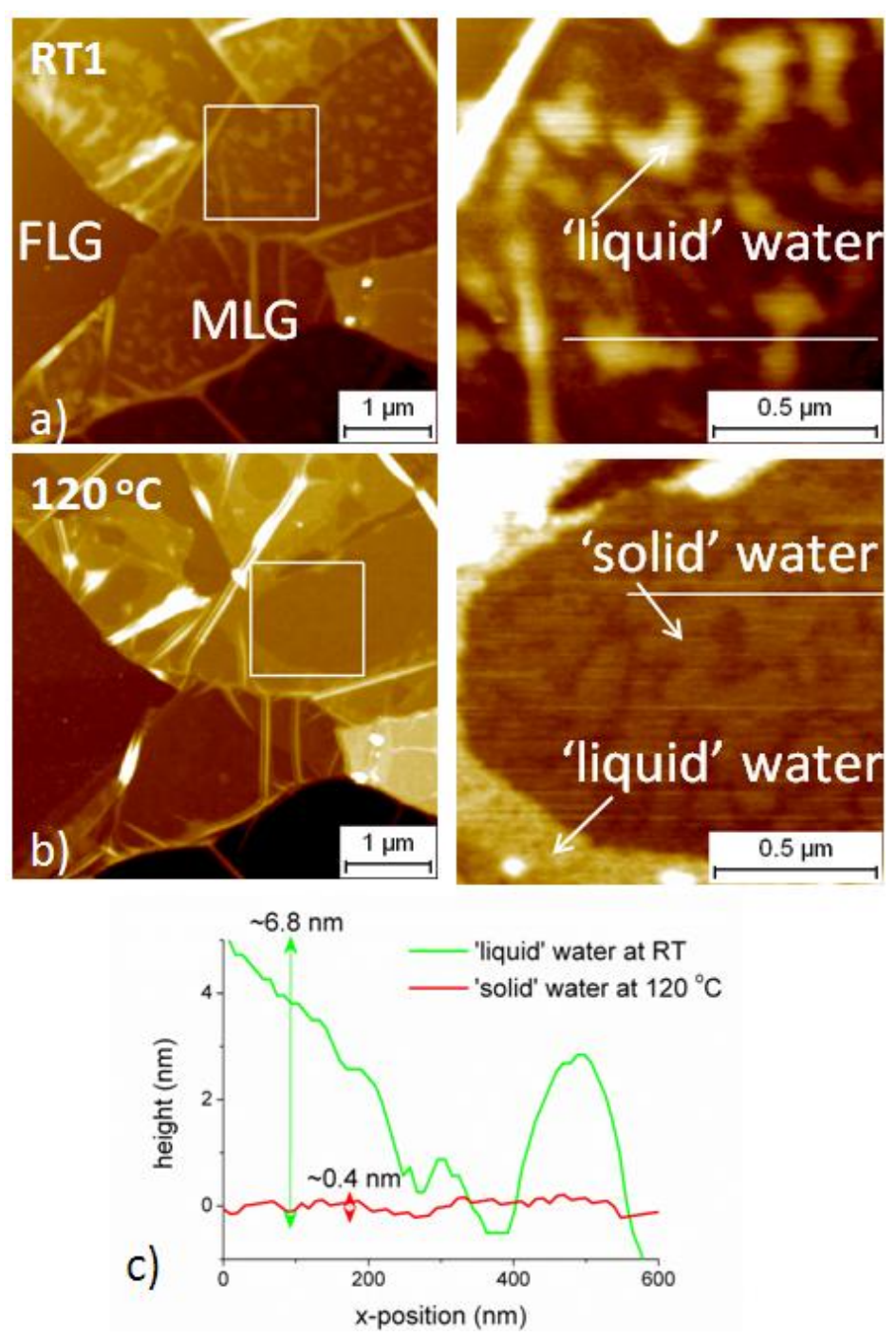

When the sample was initially scanned at room temperature (RT), a clear pattern of droplet-like features are visible on the MLG domain (Figure 5a). It should be noted that these adsorbates have a significantly different shape and are larger in size (i.e., the width of $\sim 80-230 \mathrm{~nm}$ and height of $\sim 4.0-7.0 \mathrm{~nm}$ ) than those observed on the Si-face of $\mathrm{SiC}$, i.e., sample \#1. The adsorbates can also be associated with adsorption of atmospheric water. Despite scanning of multiple areas, no such features were observed on the FLG domains. After the sample was heated at $80{ }^{\circ} \mathrm{C}$, the distribution of the adsorbates on top of MLG changed significantly. Individual "droplets" coalesced together in larger "pools" forming a meniscus type structures with the height of $\sim 1.6-3.0 \mathrm{~nm}$ attached to terrace boundaries and puckers. A few individual islands of water are still visible in the central part of the image at $80{ }^{\circ} \mathrm{C}$. These individual small islands disappear completely when the sample was heated further up to $120{ }^{\circ} \mathrm{C}$ (Figure 5b), similar to the effect seen on graphite [80]. Thus, heating the sample revealed pronounced morphological changes, which we believe are likely to be attributable to 
desorption and rearrangement of water molecules on the graphene surface [78]. After a few temperature cycles performed between RT and $120{ }^{\circ} \mathrm{C}$, no further notable changes in the distribution of the water layer on top of graphene was observed, i.e., no re-adsorption of water occurred upon cooling the sample back to RT. Hence, the process of desorption and reorganization of water on MLG is irreversible with temperature at the specified humidity. This observation is opposite to the effect for graphene grown on the $\mathrm{Si}$-face of $\mathrm{SiC}$ (see Section 2.3.1), where a complete reversibility of water adsorption was demonstrated [9].

A close-up inspection of the framed area in Figure 5a,b reveals two distinct levels of contrast associated with water, corresponding line profiles are shown in Figure 5c. Additionally to the bright peripheral contrast of $\sim 1.6 \mathrm{~nm}$ height related to the water meniscus (left bottom corner of Figure $5 \mathrm{~b}$, right column) a much weaker contrast is observed in the central part of this image. These island-type structures with much more defined geometry, flat surface and a height of $\sim 0.4 \mathrm{~nm}$ are significantly different from initial droplets of water observed at RT and can be associated with an epitaxial growth of monolayer-thick islands of water, consistent with previous studies [81,82]. Whereas both initial appearance of water droplets at RT and their transformation at elevated temperatures are attributed to the presence of liquid water, the epitaxial layer observed at $120{ }^{\circ} \mathrm{C}$ is related to the appearance of "solid" water.

The surface potential on the C-face graphene was mapped by AM-KPFM technique as a function of temperature. The KPFM image clearly reveals two main levels of contrast (Figure 6a), where the area of the lowest potential is attributed to the FLG, and the region of the higher potential corresponds to the MLG. Additionally, areas of the liquid water on MLG are seen as the brightest contrast, which, however, due to the large thickness of liquid water is attributed to the electronic properties of water itself rather than modification of the graphene surface potential. The histogram analysis of the acquired KPFM data reveals two major peaks corresponding to FLG (left) and MLG (right) domains with a potential difference of $\Delta V_{\mathrm{CPD}}=200 \mathrm{mV}$ at RT (Figure 6b). This value increases up to $\sim 260 \mathrm{mV}$ at $120{ }^{\circ} \mathrm{C}$, primarily due to the shift of the $V_{\mathrm{CPD}}$ of MLG [78].

Figure 6. (a) KPFM surface potential image of graphene on $\operatorname{SiC}(000-1)$ at RT, vertical scale is $277 \mathrm{mV}$ and (b) typical histogram of the KPFM image.
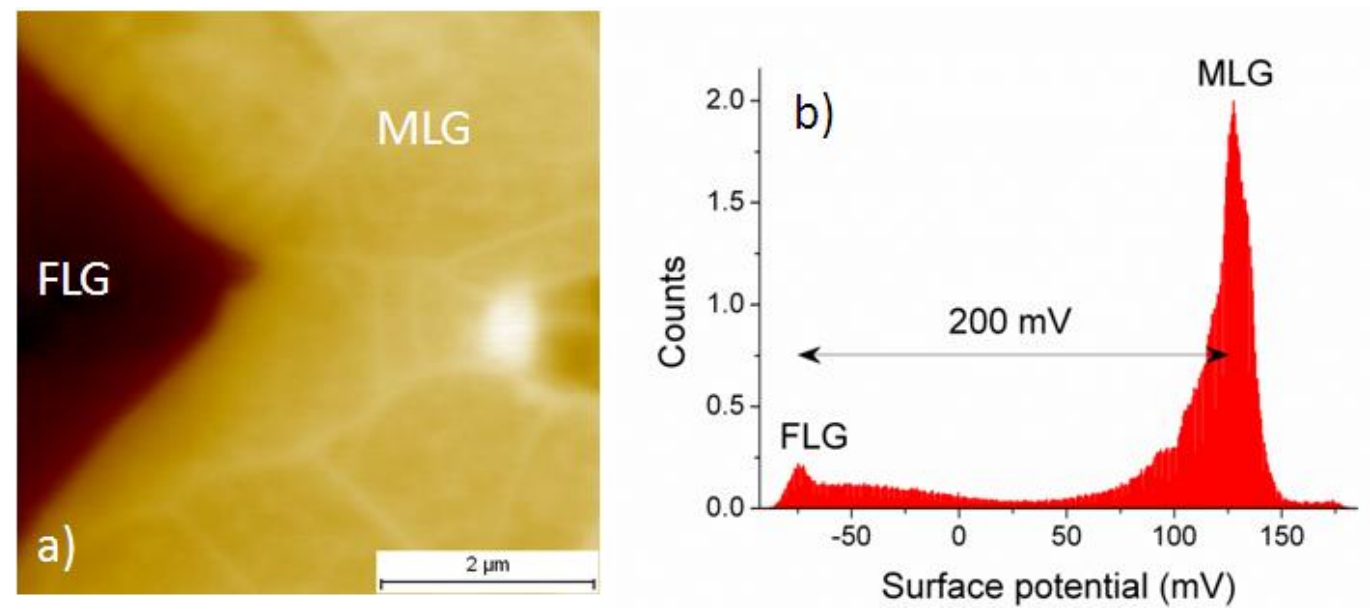
The complex morphology of the MLG domain indicates that the area consists of many domains with variable number of graphene layers. Unlike the Si-face, where growth can be effectively controlled to give large regions of uniform thickness, it is common for the C-face to have a 3D growth, i.e., obtain graphene domains of very different thickness growing next to each other [83]. The ability to control growth on the $\mathrm{C}$-face and be able to provide the same high level of uniformity as on the Si-face is still under investigation (see [84] and references therein). However, the KPFM contrast is fairly uniform within the area $(\sim 30 \mathrm{mV}$ signal variation), implying that thick graphene layers have characteristically small difference in the surface potential, which is in a good agreement with previous results on exfoliated graphene [19,21]. Thus, KPFM results confirm presence of vast areas of C-face graphene with larger thickness $(\geq 10$ layers) and demonstrate that the surface potential changes according the same rules as previously observed in the case of the exfoliated graphene and graphite.

To provide further insight into wettability of graphene domains of different thickness, we have performed microscopic contact angle measurements on bare $\mathrm{SiC}(0001)$ substrate, 1 LG on $\mathrm{SiC}(0001)$ and MLG on $\mathrm{SiC}(000-1)$. Figure 7 shows snapshots of water droplets on bare $\mathrm{SiC}\left(73.1^{\circ} \pm 1.47\right)$, IFL $\left(75.1^{\circ} \pm 1.25\right), 1 \mathrm{LG}\left(72.9^{\circ} \pm 1.27\right)$ and $\mathrm{MLG}\left(91.6^{\circ} \pm 3.05\right)$ samples. Within the experimental accuracy, IFL and 1 LG do not change wettability of the $\mathrm{SiC}(0001)$ substrate, remaining significantly less hydrophobic as previously reported [26]. However, a substantial increase of the contact angle was observed for the MLG sample on the C-face, where the measured value approaches the results typically reported in the literature for graphite. The contact angle results also support the previous observation that wettability of the substrate controls water affinity of thin graphene domains [29].

Figure 7. Contact angle measurements. Water droplet on (a) $\mathrm{SiC}(0001)$ substrate; (b) interfacial layer (IFL); (c) 1 LG and (d) MLG.

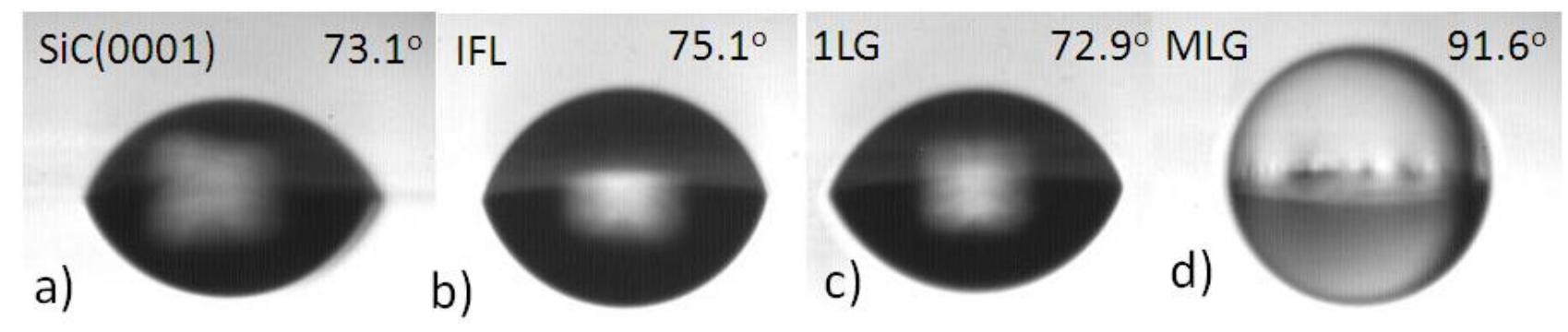

\subsubsection{Discussion}

In this section, we summarize the effect of water on the electronic properties of both types of epitaxial graphene samples grown on the $\mathrm{Si}$ - and $\mathrm{C}$-faces of $\mathrm{SiC}$ and discuss affinity of water to graphene.

In general, the $V_{\mathrm{CPD}}$ value reflects the carrier concentration and position of the Fermi energy $\left(E_{\mathrm{F}}\right)$, level in a material. Graphene grown on $\mathrm{SiC}(0001)$ is intrinsically $\mathrm{n}$-doped due to the presence of the substrate and interfacial layer [70], whereas graphene grown on $\mathrm{SiC}(000-1)$ is generally p-doped (although some degree of controversy still remains) [85-88] with the level of doping in both cases strongly dependent on a particular substrate and growth conditions (Section 2.3). Additionally, when the sample is exposed to the atmospheric environment, adsorption of water leads to the external p-doping of graphene, providing a range of rather uncontrolled doping levels [34], and promotes the 
influence of other gaseous adsorbates, such as oxygen [89]. These results were supported by molecular modeling, which takes also into account the additional factor of the orientation of the adsorbed molecule with respect to the efficacy of the charge transfer [90].

Thus, exposure to the atmospheric water at RT leads to a change of the carrier concentration (e.g., in the case of Si-face graphene, recombination of the carriers and lower $n_{\mathrm{e}}$ ) and, therefore, the Fermi energy and $V_{\mathrm{CPD}}$. As water desorbs (at least partly) at elevated temperatures, the intrinsic carrier concentrations restores, leading to a corresponding change of $V_{\mathrm{CPD}}$, as it was also observed in [21].

The observed morphological changes are fully consistent with evolution of the surface potential difference of graphene with temperature, i.e., irreversible change of $V_{\mathrm{CPD}}$ correlated with absence of re-absorbed water after thermal cycling on $\mathrm{C}$-face graphene, as opposed to the fully reversible variation of $V_{\mathrm{CPD}}$ and re-absorption of water on $\mathrm{Si}$-face graphene. For later, the temperature rise causes the water to desorb initially from thicker (i.e., $3 \mathrm{LG}$ at $50{ }^{\circ} \mathrm{C}$ ) domains, followed by $2 \mathrm{LG}$ (at $80{ }^{\circ} \mathrm{C}$ ), however no significant changes are seen in the state of 1 LG (Figures 3 and 4). A similar situation is observed in the case of C-face graphene for FLG and MLG, respectively. This observation indicates that the heat of desorption (binding energy) of water on graphene is lower for thicker graphene domains. This is supported by the fact that a (sub)monolayer of water on either 1 LG or FLG is not affected by the highest applied temperature $\left(120^{\circ} \mathrm{C}\right)$, i.e., the heat energy is smaller than the energy required to desorb the water from thin graphene, thus also implying that thin graphene is essentially hydrophilic reflecting the properties of the $\mathrm{SiC}$ substrate. General hydrophilicity of $1 \mathrm{LG}$ as shown in our macroscopic contact angle measurements confirms the fact that water is indeed present on the surface. The described experiments indicate that hydrophobicity increases with layer number in both types of graphene, such that $\mathrm{SiC} \rightarrow 1 \mathrm{LG} \rightarrow 2 \mathrm{LG} \rightarrow 3 \mathrm{LG}$ on the Si-face (bearing in mind that pristine $\mathrm{SiC}$ is hydrophilic) and FLG $\rightarrow$ MLG on the C-face. These observations obtained from nanoscale SPM measurements are in agreement with our macroscopic measurements of the contact angle performed on different types of graphene [9,78] as well as with results of others [28]. This also agrees well with the results of molecular dynamic modeling of water on epitaxial graphene [27] and is generally consistent with a value of the adsorption (binding) energy for water molecules being significantly larger on pure $\mathrm{SiC}(-636 \mathrm{meV})$ [91] than on graphene (18-47 meV) [90], though in the latter case the influence of the substrate was not taken into account.

Thus, the process of desorption of water is generally similar for both $\mathrm{Si}$ - and C-face grown graphene and governed by affinity of water to domains of different thickness. However, the re-adsorption process is significantly different. This could be explained by a strain-free state of the thick MLG on $\mathrm{SiC}(000-1)$ and the lack of one-dimensional defects within the domains, acting as effective nucleation sites for water absorption, as opposite to thin graphene layers on $\mathrm{SiC}(0001)$, where we attribute re-adsorption of water and formation of distinctive patterned structures on 2-3 LG to the presence of such defects and strain, which act as nucleation sites for the epitaxial growth of water $[38,63]$. The initial nucleation of water droplet on $\mathrm{C}$-face graphene could occur prior the experiment during uncontrolled exposure of the sample to increased humidity conditions.

It is a common belief that carbonaceous surfaces (including graphite and graphene) are hydrophobic. At the same time, the exact values of the contact angle was shown to vary significantly, i.e., $\sim 93^{\circ}-120^{\circ}$ [26-28]. Independence of the wettability of graphene on its thickness have been reported [26]. A value of the contact angle $\left(86^{\circ}\right)$ was simulated for an isolated sheet of graphene [92]. 
The substrate geometry affects the water affinity to graphene, i.e., it has been theoretically predicted that the increased roughness of the graphene might enhance the adsorption of water, providing closer proximity of the water molecule to carbon atoms [93]. On hydrophobic graphite surfaces under humid atmospheres it has been shown that water preferentially adsorbs along step edges, forming 5-15 nm thick liquid droplets with 100-300 $\mathrm{nm}$ in lateral dimensions [80]. At the same time on hydrophilic mica, water forms "epitaxial" ice-like islands of large lateral dimensions but only $\sim 0.4 \mathrm{~nm}$ thick [25].

For such hydrophilic substrates as gold and glass, it has recently been demonstrated that thin-layer exfoliated graphene only slightly modifies their wettability [29], and the reported contact angles, $\sim 33^{\circ}-48^{\circ}$, still remain significantly less than the expected value of $\sim 93^{\circ}$. While pristine, freshly prepared hydroxyl-terminated $\mathrm{SiC}$ is strongly hydrophilic, with water contact angle for Si-face surface being only $\sim 15^{\circ}$ [94], exposure to environmental conditions and chemical cleaning leads to formation of stable $\mathrm{C}-\mathrm{O}-\mathrm{C}$ bonds, which unavoidably increases hydrophobicity of the $\mathrm{SiC}$, and so the values similar to the one demonstrated in Figure 7a become rather typical. When carbon bonds appear on top of SiC (including formation of an IFL), the relative hydrophilicity decreases and the contact angle increases [94,95], though still remaining significantly less hydrophobic than in MLG or graphite. In the case of epitaxial graphene, the wetting properties of a thin graphene layer are dominated by $\mathrm{SiC}$ and the IFL (in case of $\mathrm{SiC}(0001)$ ), which essentially results in a more hydrophilic graphene than that predicted. However, as the thickness of graphene increases, its properties became more hydrophobic and approaches the values typically reported for graphite. Wettability transparency of graphene on SiC is controlled by van der Waals forces and can be described by a lack of short-range chemical bonding [29]. Thus, both SPM-derived (i.e., nanoscale) and contact angle (i.e., macroscale) results indicate that wettability of graphene depends on its thickness and the underlying substrate. Damage introduced in the graphene has also been shown to increase the wettability or hydrophilicity [26]. This indicates that defects in graphene, which may occur during growth or device fabrication process, will facilitate adsorption of atmospheric species.

On the basis of the results presented here, we stress the importance of considering graphene properties only in conjunction with those of the substrate (i.e., level of doping), which not only affects the charge transport, i.e., the carrier type and concentration, but may also lead to modification of the chemical properties of graphene, in particular its affinity to water. The other important factor is the state of the graphene surface (e.g., presence of other adsorbates, strain and defects, treatment history, resist residues, etc.), which is significantly affected by sample history, exposure to atmosphere and even storage conditions. Thus, no straightforward answer to the question about the wettability of graphene is feasible and all above considerations should be taken into account.

The evidence presented here, including the thickness of the adsorbed layer, the temperature dependence of the desorption/reabsorption process, and its consistent influence on the surface potential of the graphene due to extrinsic doping as well as wettability of graphene showing increasing hydrophobicity with number of layers and good correlation between nano- and macroscopic results, provide a convincing evidence that the adsorbed species are related to atmospheric water. 


\subsection{Electrical SPM for Studies of Graphene Devices}

At present, the stages involved in determining sheet resistance, mobility, and carrier density require a graphene sample to be lithographically patterned and electrically contacted to form a Hall bar device (Figure 8) before measurements can be made. The invasive nature of patterning and contacting steps is known to influence the results [96-99], e.g., affect the carrier balance and even type due to unavoidable presence of the resist residue. Using KPFM, the functionality of devices on the local level can be predicted and analyzed without time-consuming and expensive fabrication and measurement procedures. Despite being local measurements, these methods do allow for a wafer scale testing of the film quality by a programmable automated movement of the probe position for the recording of results at multiple locations on a wafer of devices, relevant for upscale production of graphene films and essential for industrial applications.

Figure 8. Schematic of a typical Hall bar device for transport measurements described in [97], where the current $\left(I_{\text {bias }}\right)$ is applied across the $I_{\text {source }}$ and $I_{\text {drain }}$, the Hall voltage $\left(V_{\mathrm{H}}\right)$ arising due to a magnetic field $(B)$ perpendicular to the surface is measured across $V^{+}$and $V^{-}$. The four-terminal $\left(R_{4}\right)$ resistance of the channel is measured by current biasing the device and measuring the voltage drop across $V_{1}$ and $V_{2}$, thus neglecting the contact and lead resistances. The $V_{\mathrm{H}}$ and $R_{4}$ measurements allow determination of carrier density, $n=I_{\text {bias }} B / e V_{\mathrm{H}}$, and mobility, $\mu=1 / n e R_{\mathrm{s}}$, of the material, where $e$ is the electronic charge and $R_{\mathrm{s}}$ is the graphene sheet resistance.

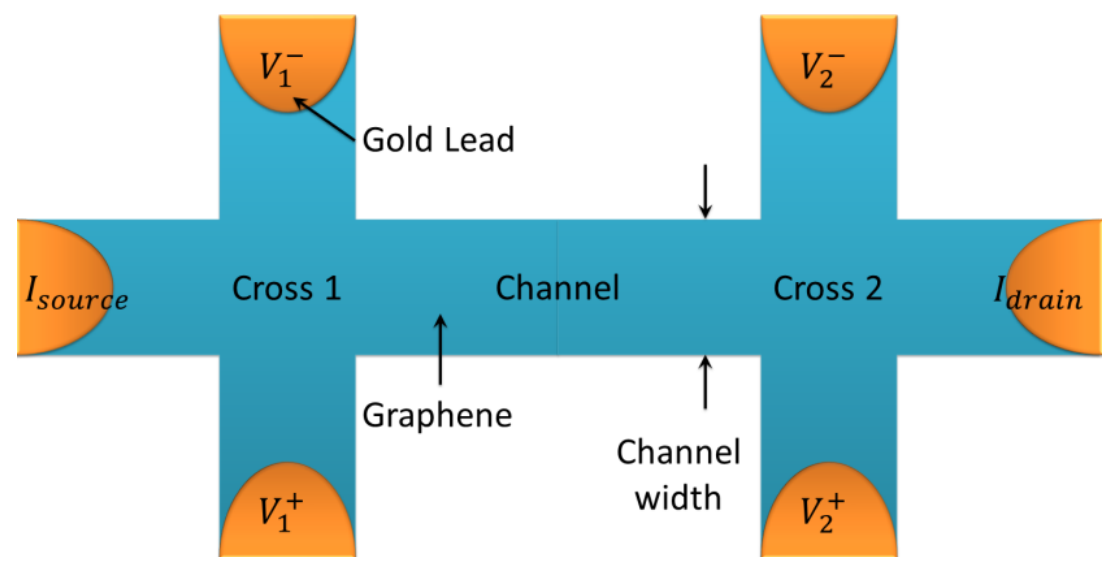

\subsubsection{Influence of External Conditions}

Substances that come into direct contact with graphene have a profound effect on its electronic properties. As previously described in Section 2.3, graphene on $\mathrm{SiC}(0001)$ is intrinsically n-doped [32,57,100]. Additionally, the high humidity level [7,25,80], gasses [87] and photochemicals $[17,101]$ attached to the surface of graphene act as sources of extrinsic doping, providing both $\mathrm{n}$ - and p-type dopants (Sections 2.2 and 2.3). While effects of humidity and gasses can be overcome by performing measurements in vacuum, substrate and photochemical effects are less easy to control. In this section, we present our investigation on the effects of resist residues left over from the electron beam lithography fabrication process, on the electronic properties of graphene devices. The 1- $\mu \mathrm{m}$ wide devices (\#3a and \#3b) studied in Sections 2.4.1 and 2.4.2 were fabricated out 
of $1 \mathrm{LG} / 2 \mathrm{LG}(\sim 96 / 4 \%)$ on $\mathrm{SiC}(0001)$. At 1-2 nm thick, the grain like nature of the residues is easily observed with AFM (Figure 9a). Prior to cleaning device \#3a, transport measurements were performed in ambient conditions revealing strong p-type behavior with $n_{\mathrm{h}}=6.61 \times 10^{11} \mathrm{~cm}^{-2}$ (Table 2). CM-AFM was then used to scrape away the residues from the measurement region of the device (Figure 9b). The device was then measured immediately after the cleaning process and five days later (whilst stored in a desiccator with $<20 \%$ relative humidity), where transport measurements revealed n-type behavior with $n_{\mathrm{e}}=2.55 \times 10^{12}$ and $n_{\mathrm{e}}=4.71 \times 10^{11} \mathrm{~cm}^{-2}$, respectively. The restored n-type conductivity of cleaned $1 \mathrm{LG}$ on $\mathrm{SiC}(0001)$ is consistent with theory and experimental work presented by various other groups $[6,102,103]$.

Figure 9. Topography images showing device \#3a, (a) before and (b) after CM-AFM cleaning; (c) The line traces for each image along the dashed white line as indicated in (a).

(a)

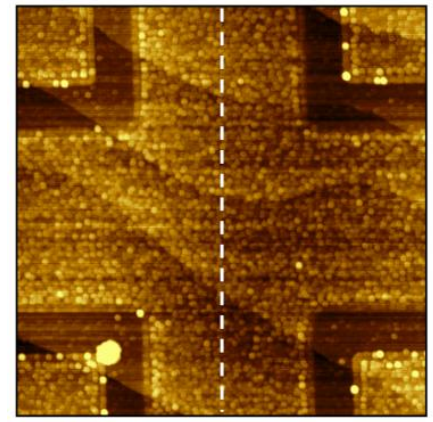

Before Cleaning (b)

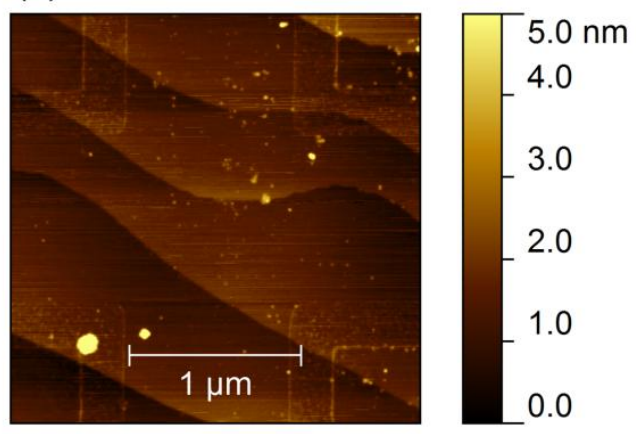

After Cleaning (c)

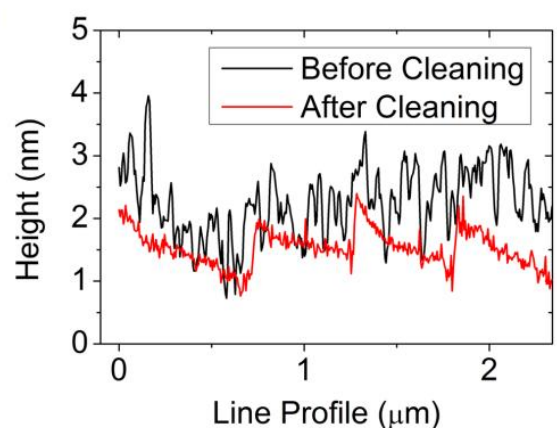

Table 2. A summary of the four-terminal resistance $\left(R_{4}\right)$, Hall coefficient $\left(R_{\mathrm{H}}\right)$, carrier concentration $(n)$ and carrier mobility $(\mu)$ for device \#3a, with residues and after CM-AFM cleaning of the residues from the lithography process.

\begin{tabular}{ccccc}
\hline State of Device \#3a & $\boldsymbol{R}_{\mathbf{4}}(\mathbf{k} \boldsymbol{\Omega})$ & $\boldsymbol{R}_{\mathbf{H}}\left(\boldsymbol{\Omega} \mathbf{T}^{-\mathbf{1}}\right)$ & $\boldsymbol{n}\left(\mathbf{c m}^{-\mathbf{2}}\right)$ & $\boldsymbol{\mu}\left(\mathbf{c m}^{\mathbf{2}} \mathbf{V}^{\mathbf{- 1}} \mathbf{s}^{\mathbf{- 1}}\right)$ \\
\hline With residue & 26 & -945 & $n_{\mathrm{h}}=6.61 \times 10^{11}$ & $\mu_{\mathrm{h}}=1449$ \\
Cleaned & 6 & +250 & $n_{\mathrm{e}}=2.55 \times 10^{12}$ & $\mu_{\mathrm{e}}=1673$ \\
Cleaned + 5 days & 33 & +1332 & $n_{\mathrm{e}}=4.71 \times 10^{11}$ & $\mu_{\mathrm{e}}=1616$ \\
\hline
\end{tabular}

The initial p-type nature of the device was a clear consequence of the standard e-beam polymer residue, i.e., a mixture of PMMA/MMA and ZEP520, remained adhered to the graphene after the lithography process. It is important to note that, in an attempt to dissolve the 1-2 $\mathrm{nm}$ thick residues in o-Xylene, the device was exposed to 250-nm wavelength UV light for a total of $20 \mathrm{~min}$. Exposing the resist to e-beam or UV light has a profound effect on the chemical structure, effectively triggering photo-induced reactions [104]. In the case of ZEP520, neutral chlorine radicals are formed, which can generate active electron acceptors, and, thus, act as a p-dopant [105]. On the other hand, PMMA/MMA is not expected to contribute to the doping as its chemical composition does not include chlorine or other acceptor-type radicals [101]. Photochemical gating has been commonly used for decreasing $n_{\mathrm{e}}$ in graphene devices with UV exposures [3,101], for example, we previously documented change of graphene conductivity from n-type to p-type, as a consequence of electron beam $(5 \mathrm{kV})$ radiation with a low dose of $\sim 6 \mu \mathrm{C} / \mathrm{cm}^{2}$ [96]. 


\subsubsection{Calibrated Work Functions of p-n Junction}

As discussed above, the presence of resist residues has a profound effect on the electronic properties of graphene, such that the conduction mechanism is hole driven. Complete removal of the resist residue from a part of the device using CM-AFM, allows us to fabricate a unique planar p-n junction on epitaxial graphene (device \#3b: left part-p-type, right part-n-type, Figure 10a,b). Transport measurements revealed cross 1 to remain p-type, while the cleaned cross 2 showed the $n$-type behavior (Table 3). For both types of doping, carrier concentration and the expected $E_{\mathrm{F}}$ were comparable.

Table 3. A summary of transport properties in ambient conditions for device \#3b, where cross 2 is cleaned of resist residues. The Hall coefficient, carrier density and work function are $R_{\mathrm{H}}, n$ and $\Phi$, respectively and the Fermi energy is $E_{\mathrm{F}}(n)=\hbar v_{\mathrm{F}} \sqrt{ }(\pi n)$, where $v_{\mathrm{F}}$ is the Fermi velocity.

\begin{tabular}{cccccc}
\hline Device \#3b & Carriers & $\boldsymbol{R}_{\mathbf{H}}\left(\boldsymbol{\Omega} \mathbf{T}^{-\mathbf{1}}\right)$ & $\boldsymbol{n}\left(\mathbf{c m}^{-\mathbf{2}}\right)$ & $\boldsymbol{E}_{\mathbf{F}}(\mathbf{e V})$ & $\boldsymbol{\Phi}(\mathbf{e V})$ \\
\hline Cross 1 (resist residue) & Holes & -250 & $n_{\mathrm{h}}=2.50 \times 10^{12}$ & 0.148 & $4.68 \pm 0.05$ \\
Cross 2 (cleaned) & Electrons & +535 & $n_{\mathrm{e}}=1.17 \times 10^{12}$ & 0.101 & $4.35 \pm 0.05$ \\
\hline
\end{tabular}

Figure 10. (a) Topography and (b) surface potential map performed using FM-KPFM showing device \#3b after CM-AFM cleaning of the right-hand-side of the device only; (c) EFS measurements performed on p-type and n-type graphene; (d) Work function versus carrier density from EFS and transport measurements.

(a)

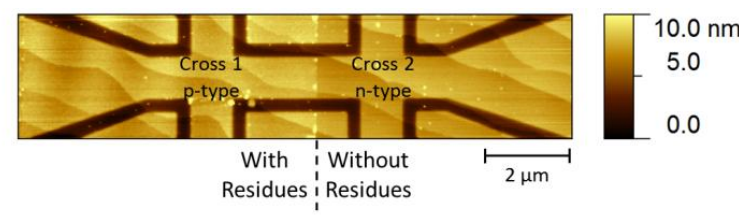

(c)

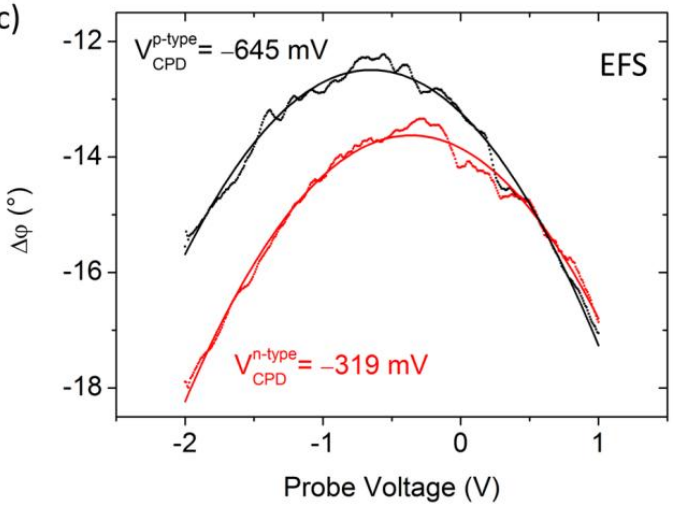

(b)

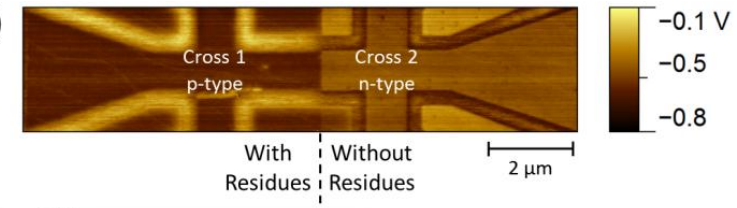

(d)

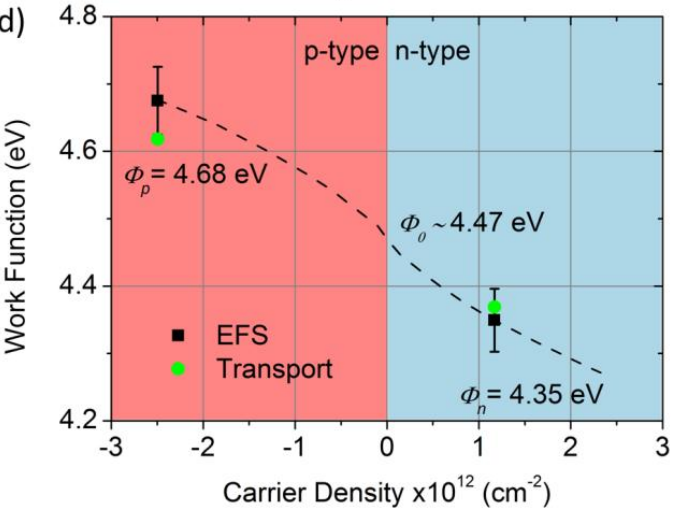

It is vital to characterize the work function of the graphene p-n junction, which was measured using EFS (Figure 10c). The work function of gold, determined to be $\Phi_{\text {gold }}=4.82 \mathrm{eV}$ by ultraviolet photoemission spectroscopy, was used as a stable reference point to which the work function was calibrated for the doped silicon probe PFQNE-AL (Bruker) with a radius of $\sim 5 \mathrm{~nm}$ and a force constant of $\sim 0.8 \mathrm{~N} / \mathrm{m}\left(\Phi_{\text {probe }}=4.03 \mathrm{eV}\right)$. The work functions of p-type and n-type graphene in device \#3b were measured as $\Phi_{\mathrm{p}}=4.68 \pm 0.05 \mathrm{eV}$ and $\Phi_{\mathrm{n}}=4.35 \pm 0.05 \mathrm{eV}$, respectively, hence, the doping due to resist residue increases the work function by $\Delta \Phi_{\mathrm{p}-\mathrm{n}}=330 \pm 100 \mathrm{meV}$. This is consistent with the Fermi 
energies calculated from transport measurements, where $\Delta \Phi_{\mathrm{p}-\mathrm{n}}=249 \mathrm{meV}$ (Figure 10d). Assuming a symmetrical S-shape dispersion [106] and plotting $\Phi$ as a function of $n$, the intrinsic work function (i.e., at the Dirac point) was extrapolated to be $\Phi_{0}=4.47 \pm 0.05 \mathrm{eV}$, which is comparable to a previously published value of $\Phi_{0}=4.57 \pm 0.05 \mathrm{eV}$ [106]. The same authors also demonstrated a $\Delta \Phi_{\mathrm{p}-\mathrm{n}} \sim 300 \mathrm{meV}$ using bottom gate voltage $\Delta V_{\mathrm{g}} \sim 60 \mathrm{~V}$ [106], however, the lack of complementary carrier density measurements prohibits a direct comparison with our results.

\subsubsection{Calibrated Work Functions of Single- and Double-Layer Graphene}

The EFM technique was used for mapping the local variations in electronic properties of a $4.8-\mu \mathrm{m}$ wide device (\#4) fabricated out of $1 \mathrm{LG} / 2 \mathrm{LG}(\sim 94 / 6 \%)$ on $\mathrm{SiC}(0001)$, which has been cleaned of residues using CM-AFM (Figure 11a). Using the latter for probe calibration in EFS measurements, 200 individual points were performed along the dashed line indicated in Figure 11a inset, which includes measurements on $1 \mathrm{LG}, 2 \mathrm{LG}$ and the gold electrodes. Again using $\Phi_{\text {gold }}=4.82 \mathrm{eV}$ as a reference, the doped silicon probe PFQNE-AL work function was determined to be $\Phi_{\text {probe }}=4.09 \mathrm{eV}$ and the work functions of $1 \mathrm{LG}$ and $2 \mathrm{LG}$ was determined to be $\Phi_{1 \mathrm{LG}}=4.55 \pm 0.02 \mathrm{eV}$ and $\Phi_{2 \mathrm{LG}}=4.44 \pm 0.02 \mathrm{eV}$, respectively, in ambient conditions (Figure 11b). The change in work function between $1 \mathrm{LG}$ and $2 \mathrm{LG}$ of $\Delta \Phi_{1-2 \mathrm{LG}}=110 \pm 21 \mathrm{meV}$ is in a good agreement with values published by others groups (Table 4). It is important to note that, although the $\Phi_{1 \text { LG }}$ of device \#4 does not exactly correlate with the $\Phi_{\mathrm{n}}$ of device \#3b, the measurements are performed on two completely different batches of samples, which have different doping levels as a result of small changes in the growth, sample fabrication processes and environmental effects as previously described in Sections 2.2-2.4.

Figure 11. (a) Line profile of the surface potential obtained by EFS along the center of the channel for device \#4, indicated by the dashed line in the inset image. The inset EFM phase image shows the device consisting of primarily 1 LG, a few 2 LG islands and gold electrodes. Note that the EFM image was taken with $V_{\text {probe }}=+2 \mathrm{~V}$, where the contrast of the individual domains is opposite to surface potential (see Figure 2); (b) Band structure of n-doped 1 LG and 2 LG that are in electrical contact with each other.

(a)

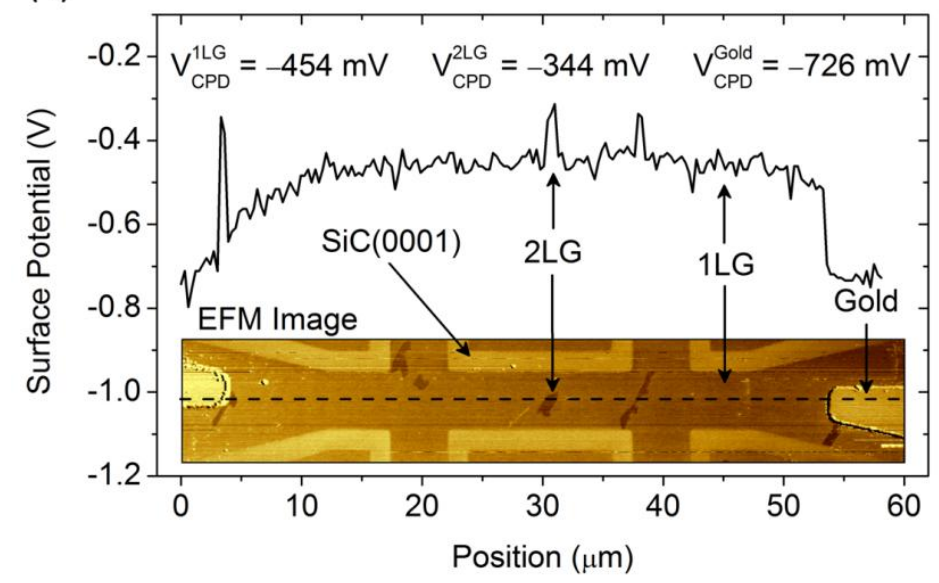

(b)

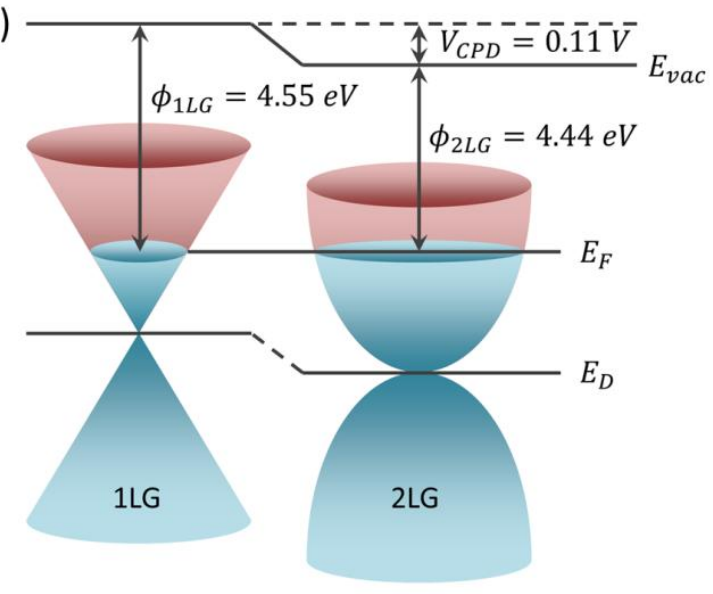


Table 4. A summary of work function measurements performed in ambient conditions.

\begin{tabular}{|c|c|c|c|c|c|}
\hline Substrate & $\Phi_{1 \mathrm{LG}}(\mathrm{eV})$ & $\Phi_{2 L G}(e V)$ & $\Delta \Phi_{1-2 \mathrm{LG}}(\mathrm{meV})$ & Technique & Reference \\
\hline $4 H-\mathrm{SiC}$ & $4.55 \pm 0.02$ & $4.44 \pm 0.02$ & $110 \pm 21$ & FM-KPFM & Device \#4 \\
\hline $4 H-\mathrm{SiC}$ & - & - & $15-50^{(1)}$ & AM-KPFM & Sample \#1 \\
\hline $6 H-\mathrm{SiC}$ & - & - & $135 \pm 9$ & FM-KPFM & [22] \\
\hline $4 H-\mathrm{SiC}$ & - & - & 35 & AM-KPFM & [107] \\
\hline Flakes on $\mathrm{SiO}_{2}$ & $4.57 \pm 0.05$ & $4.69 \pm 0.05$ & $120 \pm 50$ & FM-KPFM & [106] \\
\hline Flakes on $\mathrm{SiO}_{2}$ & - & - & 68 & AM-KPFM & {$[21]$} \\
\hline Flakes on $\mathrm{SrTiO}_{3}$ & $4.409 \pm 0.039$ & $4.516 \pm 0.035$ & $107 \pm 36^{(2)}$ & FM-KPFM & [108] \\
\hline
\end{tabular}

Note: ${ }^{(1)}$ i.e., $\Delta \Phi_{1-2 \mathrm{LG}}=15 \mathrm{meV}$ at $20{ }^{\circ} \mathrm{C}$ and $\Delta \Phi_{1-2 \mathrm{LG}}=50 \mathrm{meV}$ at $120^{\circ} \mathrm{C} ;{ }^{(2)}$ Measurements performed in ultra-high vacuum.

\section{Experimental Section}

In this section we briefly discuss all SPM techniques used to obtain quantitative and qualitative information on graphene samples. All the measurements were performed on Bruker Dimension Icon Atomic Force Microscope (www.bruker.com) [109] using IrPt-coated SCM-PIT and doped silicon PFQNE-AL probes (www.brukerafmprobes.com) [110].

\subsection{Atomic Force Microscopy (AFM)}

Atomic force microscopy forms a basis for majority of the SPM techniques providing topographical information; exploiting the van der Waals forces between the probe and sample. The pyramidal or conical probe is generally attached to the end of a silicon or silicon nitride cantilever that is free to oscillate at mechanical resonance (typically, $f_{0} \sim 50-350 \mathrm{kHz}$ ). The fundamental principle of the AFM operation uses a feedback loop to maintain the specified positive (repulsive force) or negative (attractive force) set point relating to a specified deflection of the cantilever. The deflection is measured using a laser reflected from the top side of the cantilever and onto a 4-quadrant position sensitive detector (PSD). A piezoelectric scanner maintains the deflection set point by performing $z$-direction movement, which is then recorded, thus generating the topography map.

In ambient conditions, the two principal topography AFM techniques are contact and tapping mode. Contact mode exploits the repulsive force, where the positive deflection set point is fixed and the $z$-direction movement is recorded providing atomic scale resolution imaging. Tapping mode is a variant to contact mode, where the cantilever oscillates close to $f_{0}$. Similarly, the feedback loop maintains a constant positive deflection. However, the forces between probe and sample will change the oscillation amplitude, resonant frequency and phase. The surface topography image is constructed through $z$-direction movement of the piezoelectric scanner such that constant amplitude is maintained. Topography phase imaging records the phase shift $(\Delta \varphi)$ and the amplitude $(A)$ of the cantilever's periodic oscillations relative to the cantilever drive signal (Figure 12). The $\Delta \varphi$ is a result of the energy dissipation that occurs when the probe makes contact to the sample. The energy dissipation is directly related to the changes in mechanical (a convolution of adhesion, composition, friction and viscoelasticity) and electrical properties. 
Figure 12. Change of the phase $(\Delta \varphi)$ and amplitude $(A)$ of the cantilever relative to the drive signal as a result of mechanical and electrical interactions.

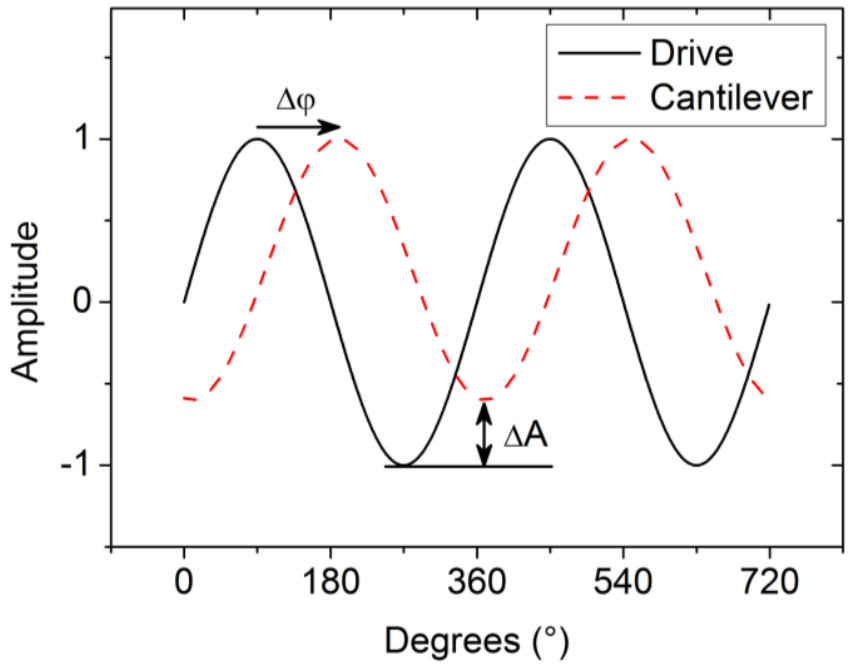

\subsection{Electrostatic Force Microscopy (EFM)}

Electrostatic force microscopy measures the electrostatic interactions between the metal coated probe and the sample. Based on a two-pass technique, the sample morphology is imaged with tapping mode AFM, the line profile of which is than retraced line by line by maintaining a constant distance between the probe and the sample (Figure 13). During the second (lift) pass, the cantilever is driven near resonance and a DC voltage bias $\left(V_{\text {probe }}\right)$ is applied to the probe with respect to the grounded sample. The sample was electrically connected to the common ground of the microscope (i.e., the sample stage) using wire bonding for devices and electrically conductive tape for as grown film samples. In our case, the experiments were typically performed at a probe-sample distance of $25 \mathrm{~nm}$ (unless stated otherwise). A potential difference between the electrically conductive probe and sample leads to electrostatic forces $\left(F=\frac{1}{2} \frac{d C}{d z} V^{2}\right)$, where $C$ and $z$ are probe-sample capacitance and probe-sample distance $[16,111,112]$. The voltage $V$ is composed of the contact potential difference $\left(V_{\mathrm{CPD}}\right)$, the applied DC voltage $\left(V_{\text {probe }}\right)$ and any other externally induced DC voltages related to an operating device ( $\left.V_{\text {induced }}\right)$, i.e., $V_{\mathrm{CPD}}+V_{\text {probe }}+V_{\text {induced. }}$ The force related to the DC bias, which deflects the cantilever, is given by

$$
\left.F_{D C}=\frac{1}{2} \frac{d C}{d z} V_{C P D}+V_{\text {probe }}+V_{\text {induced }}\right)^{2}
$$

The electrostatic forces act as a second-order effect on the mechanical oscillation and, hence similarly to tapping mode AFM, the amplitude, resonant frequency and phase are affected. The EFM image is generated by recording the cantilever phase change:

$$
\Delta \varphi=\frac{Q}{k} \frac{d F}{d z}=\frac{Q}{2 k}\left(\frac{d^{2} C}{d z^{2}}\right) V^{2}
$$


where $k$ is the spring constant and $Q$ is quality factor of the cantilever. The force gradient $(d F / d z)$ is measured with a lock-in amplifier, offering qualitative information on the electronic properties of sample surface.

Figure 13. Schematic of the EFM experimental setup. Tapping mode AFM first determines the topography of the sample, which is then traced at a constant lift height from the surface with the cantilever oscillating at the driving frequency with a DC electrical bias ( $\left.V_{\text {probe }}\right)$ applied to the cantilever.

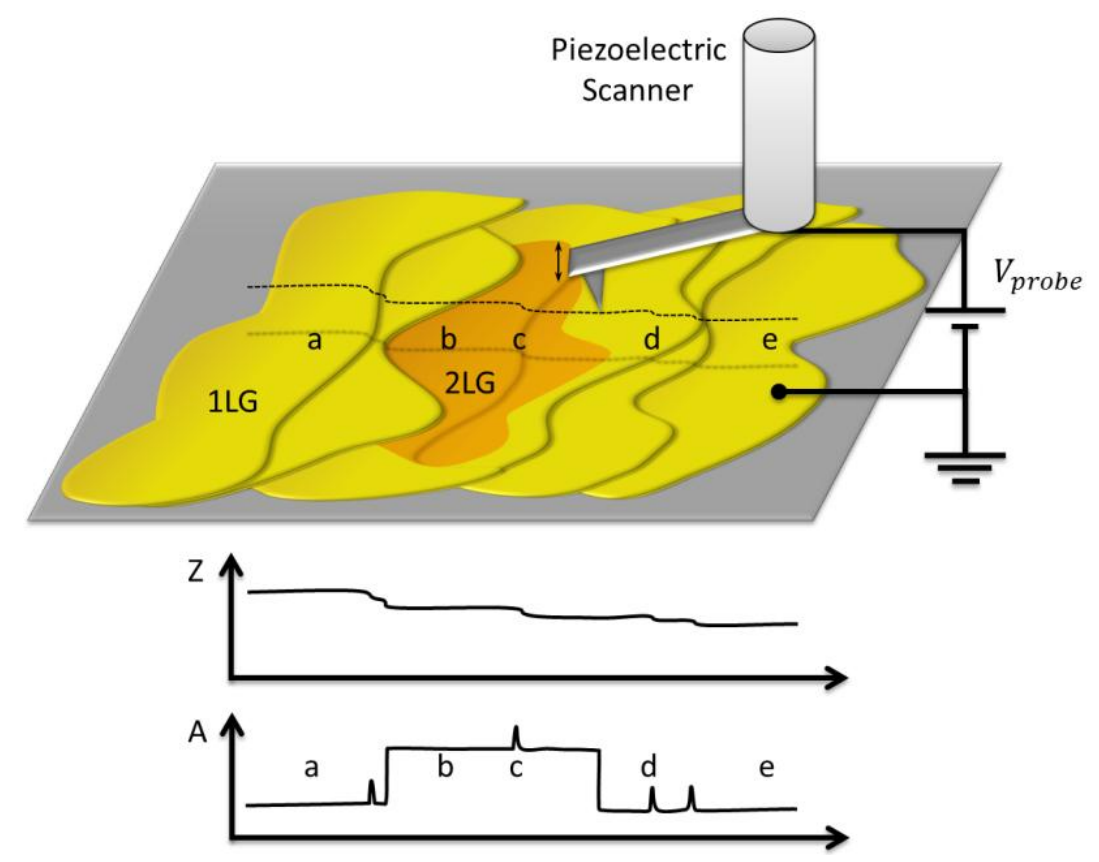

\subsection{Electrostatic Force Spectroscopy (EFS)}

Electrostatic force spectroscopy provides quantitative surface potential measurements. EFS is performed at well-defined stationary points by sweeping $V_{\text {probe }}($ i.e., -3 to $+3 \mathrm{~V}$ ) with $\mathrm{mV}$ resolution, while recording the corresponding phase change $\Delta \varphi[18,20,113]$. The $V^{2}$ term in Equation (1) leads to a parabolic response as a result of attractive and repulsive nature of separated charges (Figure 14a). The work function differences between the sample surface $\left(\Phi_{\mathrm{s}}\right)$ and the probe ( $\Phi_{\text {probe }}$ ) (Figure 15a) also constitute a $V_{\mathrm{CPD}}$ when they are in electrical contact (Figure 15b). Sweeping $V_{\text {probe }}$ determines the point which balances the electronic charges between the probe and sample (Figure 15c), effectively nullifying the force on the cantilever $(F=0)$, i.e., the value of $V_{\text {probe }}$ at the inflection point of the parabola. The raw data of the EFS was post processed using the script-based language MATLAB to extract the surface potential. EFS is the ideal method for calibrated work function measurements as it proficiently overcomes the problem of probe work function ( $\left.\Phi_{\text {probe }}\right)$ changing during scanning, which can be a major downfall of scanning probe techniques. EFM mapping is first obtained to identify the regions of interest. EFS is then performed at well-defined points, including a calibration measurement against a known quantity (i.e., gold, where $\Phi_{\text {gold }}=4.82 \mathrm{eV}$ was determined with ultraviolet photoelectron spectroscopy (UPS) measurements, Figure $14 \mathrm{~b}$ ), using $\Phi_{\text {probe }} \approx \Phi_{\text {gold }}+e V_{\text {CPD }}$ for voltage biasing the probe [114]. A doped silicon PFQNE-AL probe with a radius of $\sim 5 \mathrm{~nm}$ and a force constant 
of $\sim 0.8 \mathrm{~N} / \mathrm{m}$ has $\Phi_{\text {probe }}=4.07 \mathrm{eV}$ (Figure 14a), while a $20 \mathrm{~nm}$ IrPt coated SCM-PIT probe with a radius of $\sim 20 \mathrm{~nm}$ and a force constant of $\sim 0.8 \mathrm{~N} / \mathrm{m}$ has $\Phi_{\text {probe }}=4.99 \mathrm{eV}$. Thus, EFS offers precise and accurate quantitative measurements of $V_{\mathrm{CPD}}$ and $\Phi$. It is important to note that: traditionally, metal coated probes are used for KPFM imaging (Sections 2.1-2.3), where $\Phi_{\text {probe }}>\Phi_{\text {graphene }}$. However, a doped silicon probe was used for the measurements performed on the devices in Section 2.4; thus, $\Phi_{\text {probe }}<\Phi_{\text {graphene, }}$ which will result in a negative $V_{\text {CPD }}$. Regardless of the $\Phi_{\text {probe }}$, if for example $V_{C P D}^{2 L G}>V_{C P D}^{1 L G}$ (i.e., 2 LG is brighter than $1 \mathrm{LG}$ in KPFM images), the $\Phi_{1 \mathrm{LG}}>\Phi_{2 \mathrm{LG}}$.

Figure 14. (a) Example of a typical electrostatic force spectroscopy measurement performed on gold with a doped silicon PFQNE-AL probe. The blue and red lines represent forward (trace) and reverse (retrace) sweeps of the $V_{\text {probe }}$. The arrow points to the inflection point, where the probe voltage is the $V_{\mathrm{CPD}}$; (b) Binding energy curve for gold obtained through UPS measurements: $h=$ energy of the incident photon and $x=$ cut off binding energy determine the work function.

(a)

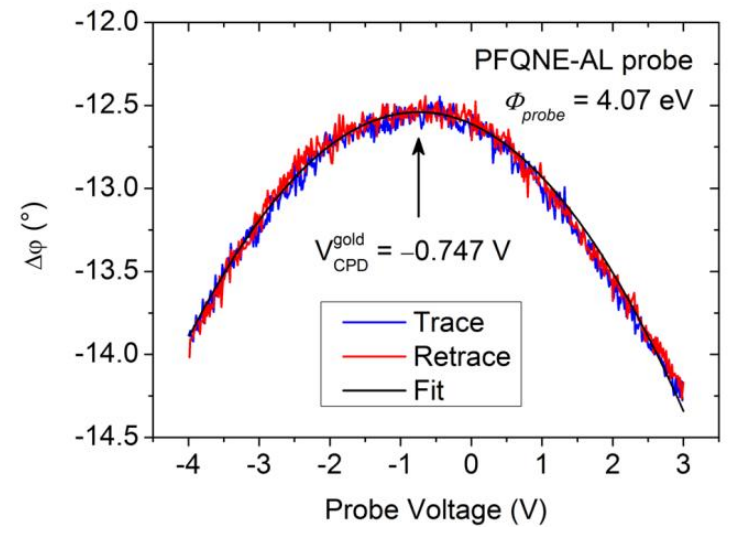

(b)

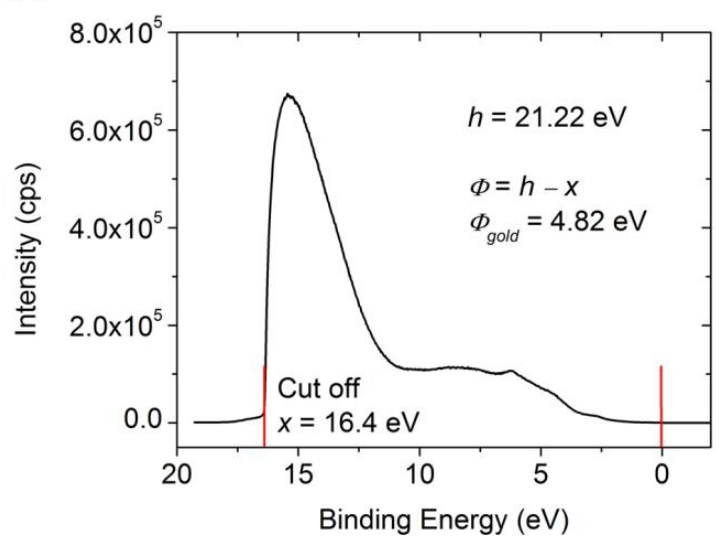

Figure 15. Scheme of energy levels for sample and doped silicon PFQNE-AL probe when they are: (a) electrically separated; (b) in electrical contact and (c) $V_{\text {probe }}$ applied to the probe to nullify the $V_{\mathrm{CPD}}$ and, thus the probe-sample electrostatic interaction. $E_{\mathrm{vac}}$ is the vacuum energy level. $E_{\mathrm{F}}^{\text {probe }}$ and $E_{\mathrm{F}}^{\mathrm{S}}$ are Fermi energy levels of the probe and gold, respectively. Note: in the case of ground connected to the sample, negative $V_{\text {probe }}$ is applied to nullify $V_{\mathrm{CPD}}$. Figure is adapted from [114].

(a)

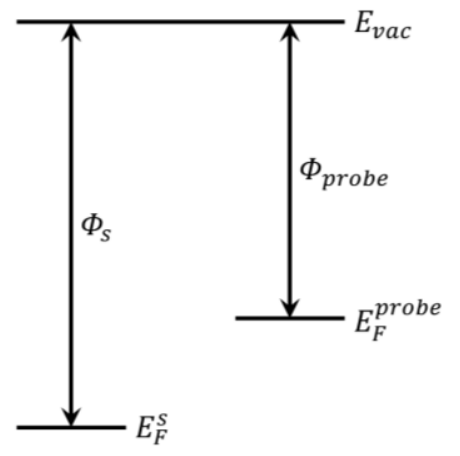

(b)

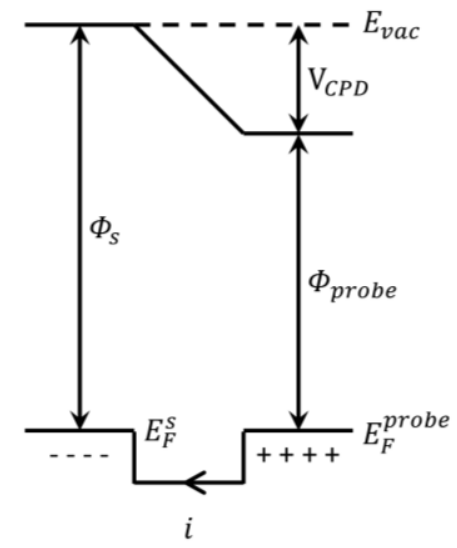

(c)

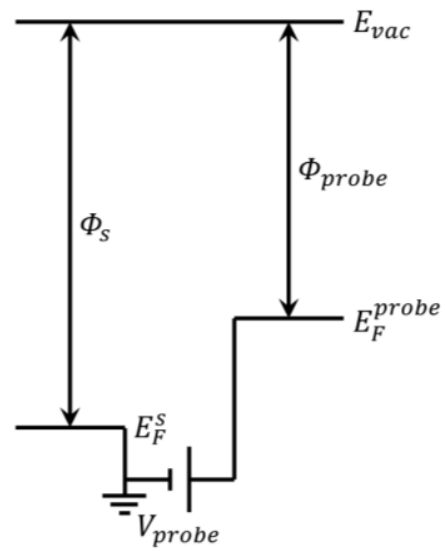




\subsection{Amplitude-Modulated Kelvin Probe Force Microscopy (AM-KPFM)}

AM-KPFM is one of the most widely used non-invasive surface potentials mapping technique (see e.g., [17] and [114]. In general, the method can be performed both as a single- and double-pass technique. In the former case, the first resonant frequency of the cantilever is used for the height and the second one for the potential measurements. The general limitation of the approach is in the low intensity of the second resonant peak and the restrictions applied by the bandwidth of the photodiode [115]. Sequentially, a two-pass technique AM-KPFM is the most common modern variety of the method (also used in the present paper), where the first pass determines the topography with tapping mode AFM and the second pass is performed with the probe lifted a set distance from the sample surface (Figure 16). In AM-KPFM the cantilever is not driven mechanically, instead AC voltage $\left(V_{\text {mod }} \sim 2 \mathrm{~V}\right.$ at $\left.f_{0}\right)$ is applied to the probe. The potential differences between the probe and sample coupled with the introduction of an AC voltage leads to mechanical oscillations in the cantilever. The AM mode is the force sensitive technique, where the force at the first harmonic is given by

$$
F_{\omega}=\frac{1}{2} \frac{d C}{d z}\left(V_{\text {probe }}-V_{C P D}+V_{\bmod } \cos 2\left(\pi f_{\bmod } t\right)\right)^{2}
$$

The AM-KPFM feedback loop nullifies $F_{\omega}$ by applying and recording $V_{\text {probe }}$ to generate the surface potential map. AM-KPFM typically requires soft cantilevers capable of large mechanical oscillations, such that the PSD can reliably detect the oscillations. This technique offers a relatively poor spatial resolution of 50-70 $\mathrm{nm}$ as a result of strong parasitic capacitance effects related to the large area of the cantilever [17].

Figure 16. Schematic of AM-KPFM experimental setup. Tapping mode AFM first determines the topography of the sample, which is then traced at a constant lift height above the surface with the AM-KPFM feedback enabled.

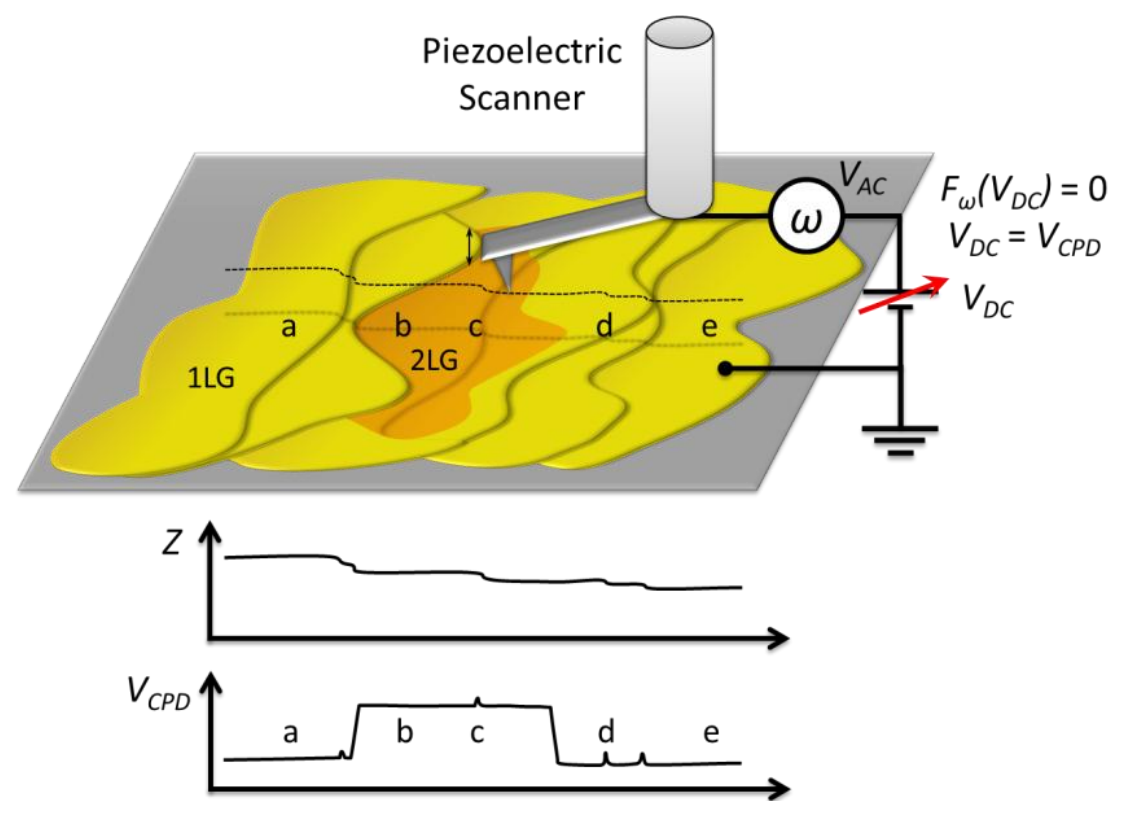




\subsection{Frequency-Modulated Kelvin Probe Force Microscopy (FM-KPFM)}

FM-KPFM operates by detecting the force gradient $(d F / d z)$ [14], which results in changes to the resonance frequency of the cantilever

$$
f_{0} \pm f_{\mathrm{mod}} \approx f_{0}\left(1-\frac{1}{2 k} \frac{d F}{d z}\right)
$$

The topography is determined with tapping mode at $f_{0}$. Simultaneously, a lower frequency $\left(f_{\text {mod }} \sim 2-8 \mathrm{kHz}\right)$ AC voltage is applied to the probe $\left(V_{\text {mod }} \sim 5-8 \mathrm{~V}\right)$ inducing frequency shift of $f_{0} \pm f_{\text {mod }}$ (Figure 17). The FM-KPFM feedback loop monitors these side modes at $f_{0} \pm f_{\text {mod }}$ and eliminates them by applying an offset voltage $V_{\text {probe }}$. In the most common variety, FM-KPFM is a single pass technique (as also performed in all relevant measurements in the present paper), though interesting examples of using it in a lift scan mode have been demonstrated recently [116]. In a similar fashion to AM-KPFM, $V_{\text {probe }}$ is recorded to generate the surface potential map. In contrast to AM-KPFM, FM-KPFM typically requires stiffer, higher frequency cantilevers. FM-KPFM offers a higher spatial resolution $(<20 \mathrm{~nm})$ as a result of detecting the electrostatic force gradient by the frequency shift $[15,21,117]$. The force gradient is highly localized to the probe apex as a consequence of short-range detection, thus, the diameter of the probe is the limiting factor of the FM-KPFM spatial resolution [114]. Likewise, the spatial resolution of EFS is also limited by the diameter of the probe as a result of detecting force gradient by the phase shift.

Figure 17. Cantilever resonance frequency $\left(f_{0}\right)$ plot showing side modes at $f_{0} \pm f_{\text {mod }}$ as a result of an AC voltage $\left(V_{\text {mod }}\right)$ applied to the probe.

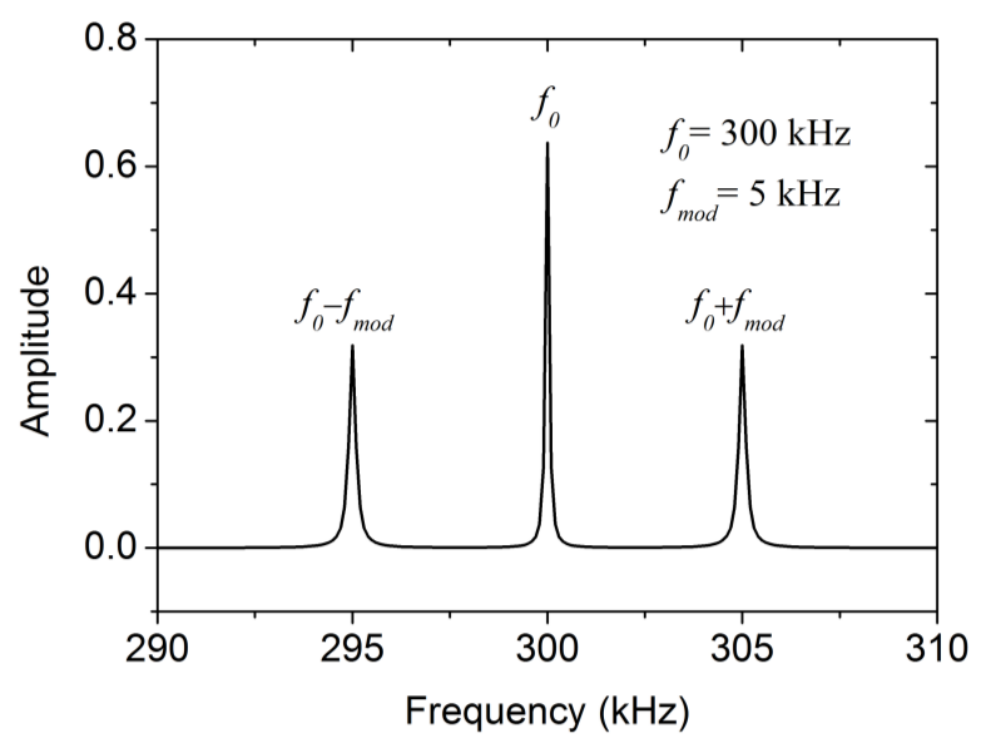

\subsection{Environmental Conditions}

All images were collected in air at temperatures $T=20-120{ }^{\circ} \mathrm{C}$ and at relative humidity of $\sim 35 \%$. For the heating experiments the graphene sample was initially placed on a large hot plate, where it was kept at a fixed temperature for one hour. The sample then was transferred to the AFM, where it was placed on the heated stage to maintain the temperature during the imaging process. This procedure 
allowed for efficient evaporation of water from the sample surface and at the same time prevented condensation of water vapor on the cantilever, preserving its mechanical and electrical properties. Imaging was performed at the following sequence of temperatures: $20,50,80,120^{*}, 80,50$ and $20^{\circ} \mathrm{C}$. At $120^{*}$ the measurement temperature was $80^{\circ} \mathrm{C}$ immediately after heating the sample to $120{ }^{\circ} \mathrm{C}$.

\subsection{Growth of Graphene Films and Raman Spectroscopy Characterization}

The one-two layer epitaxial graphene was prepared on the Si-terminated face of a nominally on-axis $4 \mathrm{H}$-SiC(0001) substrate. The substrate was pre-treated using the standard RCA cleaning procedure prior to the $2000{ }^{\circ} \mathrm{C}$ annealing at 1000 mbar argon gas pressure in a sublimation furnace. The high temperature decomposes the $\mathrm{SiC}$ layer-by-layer causing the $\mathrm{Si}$ to sublime, leaving behind the $\mathrm{C}$, which then rearranges to form the graphene honeycomb lattice. Further details of the fabrication and structural characterization are reported elsewhere [118]. The specific synthesis route has been developed to provide large areas of homogeneous graphene layers $[4,10]$. The resulting material is n-doped, owing to charge transfer from the interfacial layer, with the measured electron concentration in the range $6-20 \times 10^{11} \mathrm{~cm}^{-2}$ and mobility of $\sim 3000 \mathrm{~cm}^{2} \mathrm{~V}^{-1} \mathrm{~s}^{-1}$ at room temperatures [3,97]. In the case of $\mathrm{C}$-face grown graphene, it was grown on a nominally on-axis $\mathrm{SiC}$ substrate. The $4 \mathrm{H}$-SiC(000-1) single crystal substrate with a miscut of $\sim 0.06^{\circ}$ was also pre-treated using the standard RCA cleaning procedure prior to introduction into the sublimation furnace. The annealing was carried out at a temperature of $1800{ }^{\circ} \mathrm{C}$ with a buffer inert gas at pressure of 500-850 mbar and growth time of 15 min was used due to the increased growth rate [119]. The thickness, morphology, and electronic structure of the graphene samples were investigated using low-energy electron microscopy and diffraction, x-ray photoelectron emission microscopy and photoelectron spectroscopy.

Typical Raman spectra obtained after multiple measurements in different locations on 1-2 LG sample grown on $\mathrm{SiC}(0001)$ (sample \#1) and MLG sample (sample \#2) grown on $\mathrm{SiC}(000-1)$ are shown in Figures 18 and 19, respectively. The laser excitation length used in our Raman studies is $514 \mathrm{~nm}(2.41 \mathrm{eV})$. The original spectra are heavily dominated by $\mathrm{SiC}$ bands at $1400-2000 \mathrm{~cm}^{-1}$ (Figure 18a and 19a). In order to eliminate this background, a pristine SiC substrate was measured and its spectrum was subtracted from the graphene spectra. The resulting graphene spectra are shown in Figures $18 \mathrm{~b}$ and $19 \mathrm{~b}$. Characteristic $\mathrm{G}$ and $2 \mathrm{D}$ bands corresponding to double-degenerate $\mathrm{E}_{2 \mathrm{~g}}$ phonon modes at the center of the Brillouin zone (characteristic for $\mathrm{sp}^{2}$ carbon hybridization) and electron-phonon scattering processes, respectively, can be readily seen. Additionally, weak defect-induced (D) and inter-valley scattering $\left(\mathrm{G}^{*}\right)$ bands are detected. Combination of $\mathrm{D}, \mathrm{G}$ and $2 \mathrm{D}$ peak parameters are known to provide the necessary structural information regarding the number of graphene layers, crystalline disorder, stress, etc. [30,32,37,38,120-122]. However, some of the rules outlined for the exfoliated graphene are not that applicable to its epitaxial form (e.g., ratio of 2D/G peak intensity for determination of the layer thickness). 
Figure 18. Representative Raman spectra recorded on epitaxial graphene grown on $\mathrm{SiC}(0001)$. (a) Original spectra of 1 LG, 2 LG and SiC substrate. Graphene spectra are strongly dominated by substrate peaks; (b) 1 LG and 2 LG spectra obtained after subtraction of the SiC signal.

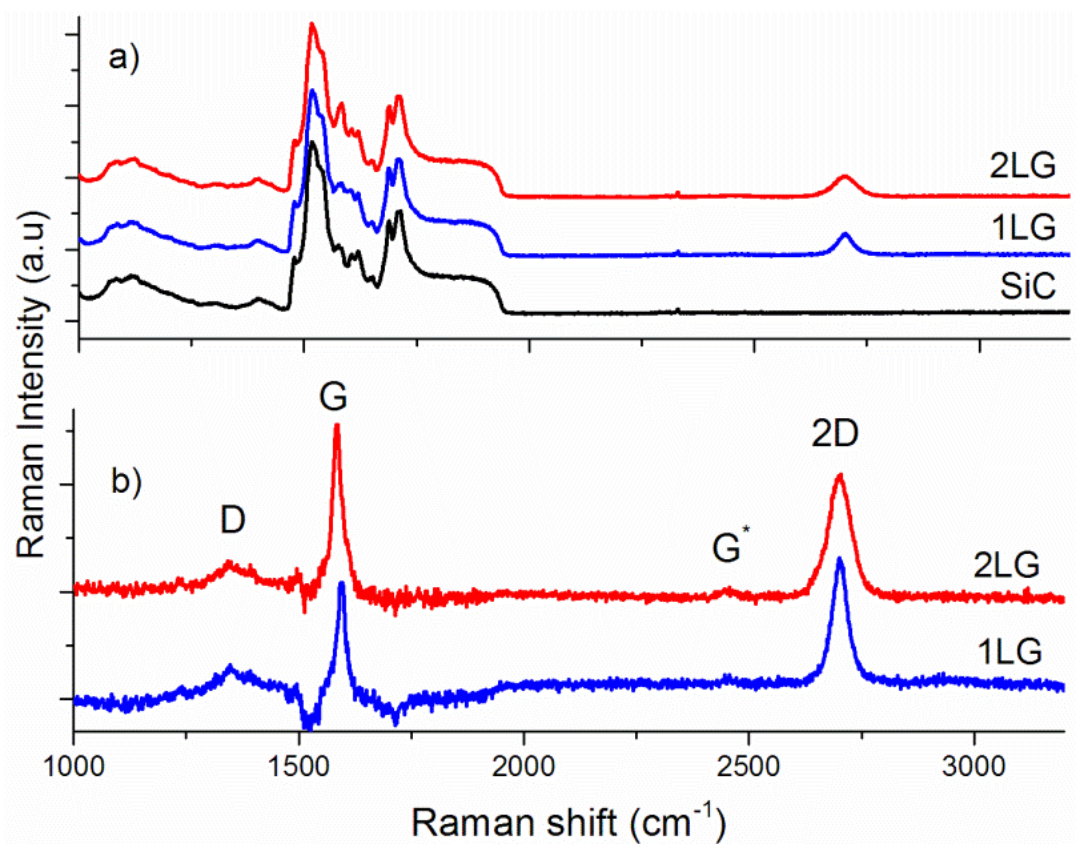

For graphene grown on the Si-face of SiC (sample \#1), two typical types of spectra are shown. While the position of the peaks is relatively unchanged, i.e., $\omega_{\mathrm{G}}=1594 \mathrm{~cm}^{-1}$ and $\omega_{2 \mathrm{D}}=2702 \mathrm{~cm}^{-1}$, the FWHM $\Gamma_{2 D}$ is significantly different in two cases, being either $\sim 40$ or $64 \mathrm{~cm}^{-1}$ (Figure 18b). This allows us to assign the narrow peak as belonging to domains of $1 \mathrm{LG}$ and the broader peak to $2 \mathrm{LG}$ in accordance to the previous Raman studies of graphene on Si-terminated face of SiC [123]. The observed shift in the G-band frequency with respect to the graphite value $\left(1582 \mathrm{~cm}^{-1}\right)$ indicates strain in the epitaxial layer grown on $\mathrm{SiC}$, which is also supported by the shift in the $2 \mathrm{D}$ band. In both cases depicted in Figure 18, the 2D band can be fitted by a single Lorentzian peak, which in case of 2 LG potentially indicates randomly rotated layers due to the weak interlayer interaction [38], as also observed on $\mathrm{CO}_{2}$-laser induced growth of graphene on $\mathrm{SiC}(0001)$ [120]. The use of an empirical formula for the 2D band [121] also confirms the correct assignment of 1 LG and 2 LG. It should be noted, that in the case of samples \#3 and \#4 2 LG could be conventionally fitted by 4 Lorentzian peaks.

For MLG grown on the C-face of SiC (sample \#2), the Raman spectra revealed a much broader 2D band with $\Gamma_{2 \mathrm{D}} \sim 70-92 \mathrm{~cm}^{-1}$ at $\omega_{\mathrm{G}}=2730 \mathrm{~cm}^{-1}$. However, this spectrum does not resemble the Raman spectrum of bulk HOPG, which is shown in Figure 19b for comparison. In particularly, the asymmetric shoulder typical for HOPG was not observed in MLG. While in this case it is difficult to define the exact number of graphene layers from Raman measurements, it is obvious that the sample is relatively thick and its defect structure is not specifically pronounced. It should be noted that the method applied above for identification of the layer thickness $[120,123]$ is not applicable to the C-face grown material. 
Figure 19. Representative Raman spectra recorded on epitaxial grown on $\mathrm{SiC}(000-1)$ and HOPG. (a) Original spectra of MLG and SiC. Graphene spectrum is strongly dominated by substrate peaks; (b) MLG spectrum obtained after subtraction of the SiC signal. HOPG spectrum is shown for comparison.

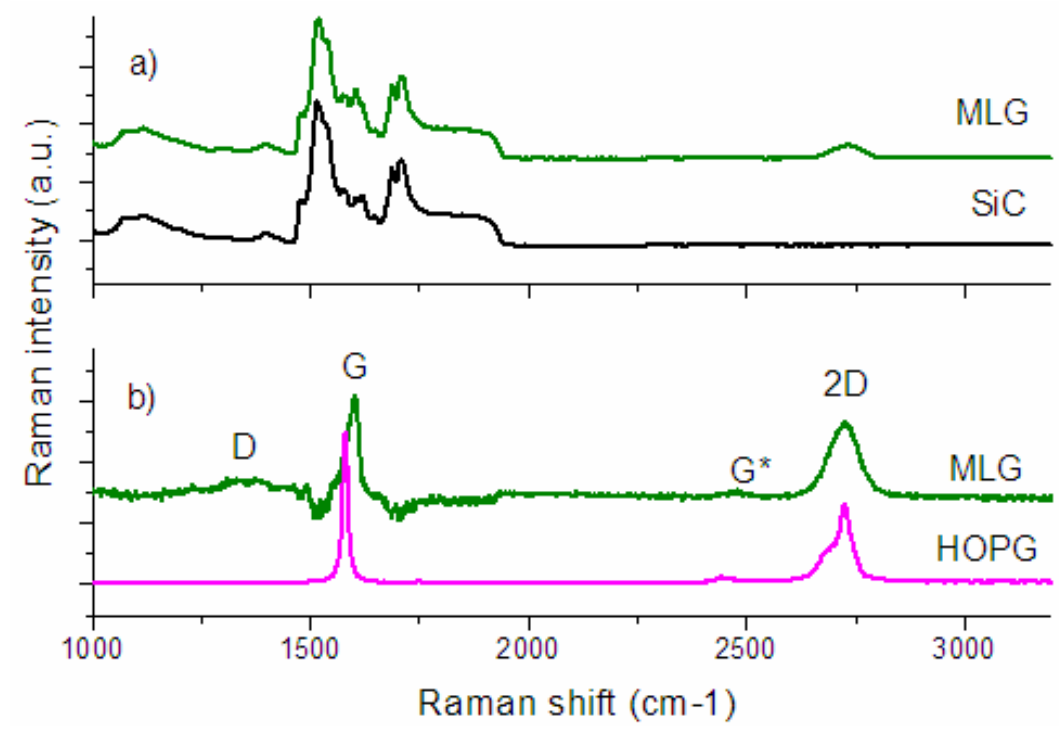

\subsection{Layer Identification}

Composition (with respect to the layer thickness) of as grown graphene samples have been confirmed with Raman Spectroscopy as described above. For all Si-face samples discussed in this work (i.e., samples \#1, \#3 and \#4) Raman confirmed the presence of 1 LG and 2 LG as the vastly predominant phases present. Subsequent EFM and KFPM analysis confirmed that generally only two levels of contrast could be identified with rare and very small domains ( $2 \%-3 \%$ of total area) of thicker graphene sometimes being found. Often areas including thicker domains of graphene were specifically chosen as regions of analysis to provide further comparison, although such regions were the exception rather than the rule. The stable step-by-step growth conditions on the Si-face combined with confirmation of the potential series for successively thicker graphene layers (e.g., [19]) allows us to confidently assign a label of $3 \mathrm{LG}$ to the regions of thicker graphene in these samples. For the C-face samples, the same process was applied, although is a less strict sense, as we only determine between FLG and MLG and the exact thickness of the layers is not explicitly known. So, despite a lack of co-localized measurements involving Raman and SPM, knowledge of the growth conditions and the expected morphology in addition to confirmation of the existing phases given by Raman provides a solid starting point.

The repeatability of the KFPM measurements is discussed with respect to a comparison of the potential difference between 1 LG and 2 LG measured on different days. For example, we show that the standard deviation of the $\Delta V_{\mathrm{CPD}}$ value is $<7 \%$ and $2 \%$ as measured at 20 and $50{ }^{\circ} \mathrm{C}$, respectively, even without taken into account the state of the probe (note, that in the former case the high uncertainty value is due to significantly smaller number of initial images obtained from the same area (as depicted in Figure 4a) at $20{ }^{\circ} \mathrm{C}$ ). This can be seen to be highly consistent but several aspects need to be controlled to achieve this, including the recent history of the sample, i.e., the sample should have 
been left to stabilize at given temperature and the measurement environment for at least an hour (ideally more). Also, quantification of the relative values is possible providing that the condition of the probe and the measurement parameters are not changed. Absolute quantification of the surface potential $V_{\mathrm{CPD}}$ requires calibration of the probe and where this is not possible, only relative values $\Delta V_{\mathrm{CPD}}$ can be confidently compared. One example of how quantification of $V_{\mathrm{CPD}}$ can be achieved is shown in Section 2.4.2 where grounded gold leads are used to provide a reference.

\subsection{Device Fabrication}

The fabrication process involved various steps of electron beam lithography (EBL), oxygen plasma etching and thermal deposition.

(1). Bonding pads: PMMA/MMA (250 $\mathrm{nm})$ and ZEP520 $(200 \mathrm{~nm})$ resist was spin coated and baked on the epitaxial graphene sample followed by EBL to define the bonding pads. The sample was developed in o-Xylene (96\%) and $\mathrm{H}_{2} \mathrm{O} / \mathrm{IPA}$ (7:93 ratio) followed by oxygen plasma to etch the graphene. $\mathrm{Cr} / \mathrm{Au}(5 / 100 \mathrm{~nm})$ was thermally evaporated followed by lift-off in acetone, forming the bonding pads with excellent adhesion to the substrate.

(2). Leads: The leads were defined in similar fashion to step 1, however with the crucial absence of oxygen plasma etching step to ensure good electrical contact to the graphene.

(3). Hall bars: PMMA/MMA (250 nm) and ZEP520 (200 nm) resist was spin coated and baked on the sample followed by EBL to write the Hall bar pattern in the resist. Developing the sample in o-Xylene (96\%) and $\mathrm{H}_{2} \mathrm{O} / \mathrm{IPA}$ (7:93 ratio) exposed the unwanted regions of the graphene while leaving behind the resist in the shape of Hall bars and leads (Figure 20). Oxygen plasma was used to etch away the unwanted graphene, leaving behind only the protected graphene to form devices with the cross width ranging from 0.5 to $5 \mu \mathrm{m}$. The remaining resist was exposed to deep UV light $(250 \mathrm{~nm})$ and dissolved, leaving behind 1-2 $\mathrm{nm}$ thick layers of resist residues.

Figure 20. Optical image of device \#4 showing the gold leads and the patterned resist protecting the graphene underneath it. The image was taken prior to the removal of the resist but after the oxygen plasma etching process.

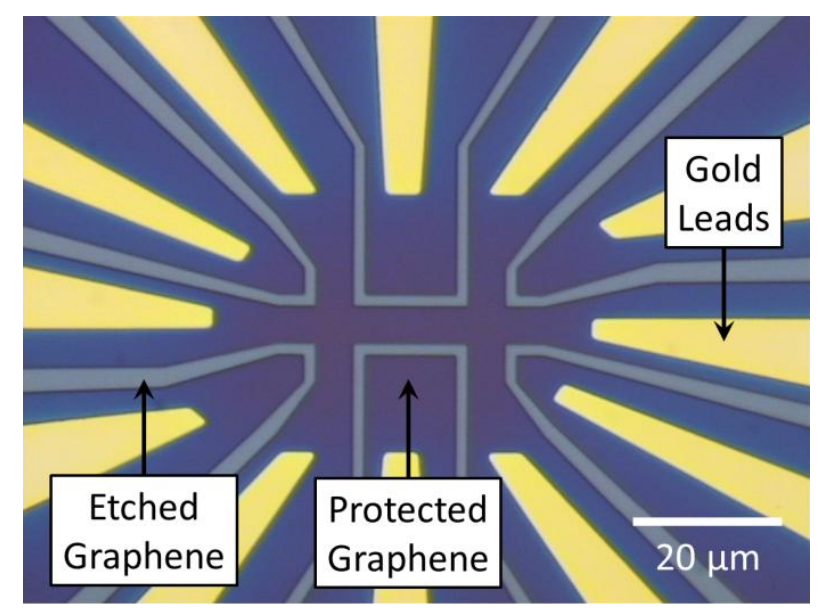

We have also estimated the contact resistance $\left(R_{\text {cont }}\right)$ between the gold leads and graphene from two- $\left(R_{2}=81.8 \mathrm{k} \Omega\right)$ and four-terminal $\left(R_{4}=23.2 \mathrm{k} \Omega\right)$ resistances of device \#4. By extrapolating $R_{4}$ to 
estimate the resistance of the graphene channel up to the gold leads $\left(R_{\mathrm{ext}}=59.3 \mathrm{k} \Omega\right)$ and assuming the resistance of the gold leads is negligible in comparison to the total resistance, we can take the difference between $R_{2}$ (includes $R_{\text {cont }}$ ) and $R_{\text {ext }}$ (excludes $R_{\text {cont }}$ ) to give an estimate of $R_{\text {cont }}=11.3 \mathrm{k} \Omega$ for each contact assuming an Ohmic behavior.

\section{Conclusions}

In this review we presented some of the recent progress related to the use of functional electrical modes of SPM for studies of graphene and graphene-based devices.

We showed that in the case of epitaxial graphene, layer determination with AFM-based topography measurements is difficult to interpret due to the complex morphology of the SiC substrate and presence of environmental adsorbates. Use of accurate height measurements and their analyses by the histogram method, coupled with a correction due to a layer adsorbed to the 1 LG, allows a very good accuracy and agreement with the theory. At the same time, functional electrical modes of SPM (i.e., EFM and KPFM) allow for a straightforward identification between domains of different thickness. In particular, quantitative KPFM measurements allow for direct measurements of the surface potential and, through calibration of the probe, the work function in graphene domains of different thickness, potentially providing a contactless method for determination of the total carrier concentration, taking into account both intrinsic and extrinsic factors influencing the carrier density.

Electronic properties as well as chemical reactivity of graphene are strongly affected by substrate interactions and exposure to the local molecular environment (i.e., water vapor, oxygen and other adsorbates). Using a combination of topography, tapping phase and surface potential studies, we investigate the temperature-dependent process of desorption and re-adsorption of water molecules on epitaxial graphene grown on $\mathrm{Si}$ - and $\mathrm{C}$-face $\mathrm{SiC}$ substrates. We demonstrate that desorption of water molecules at high temperature is accompanied by an increase in the surface potential related to the reduction of the extrinsic p-doping and shift of the overall carrier balance, thus, making the intrinsic doping a dominating mechanism. We believe that in situ observation of water evolution can potentially provide a direct method for measurements of the heat adsorption on the nanoscale. Temperature-dependent dynamics of water transformation evidently demonstrates that affinity of graphene to water depends on the layer thickness, where graphene domains with larger number of layers are more hydrophobic than thinner layers. These nanoscale results are complemented by macroscopic contact angle measurements. Thus, affinity of water to graphene should be always considered taking into account such essential aspects, as production method, type of substrate and number of graphene layers.

Furthermore, we apply functional electrical microscopies to studies of epitaxial graphene devices. For resist-free graphene devices in ambient conditions, work function measurements on n-type 1 LG and $2 \mathrm{LG}$ revealed $\Phi_{1 \mathrm{LG}}=4.55 \pm 0.02 \mathrm{eV}$ and $\Phi_{2 \mathrm{LG}}=4.44 \pm 0.02 \mathrm{eV}$, respectively, using the probe calibrated against gold electrodes. The difference of $\Delta \Phi_{1-2 \mathrm{LG}}=110 \mathrm{meV}$ is in a good agreement with values obtained by other techniques. Additionally, we show that polymers commonly used in e-beam lithography processes readily attach to graphene and form a stable residue film of 1-2 nm thickness. Such UV-modified polymer film acts as a soft gate, which can lead to a complete change of the carrier type (from n-to p-type). Based on this gating, we were able to fabricate the first planar p-n junction 
made of epitaxial graphene. In particular, we demonstrate that the work functions of $\mathrm{p}$ - and n-type graphene is $\Phi_{\mathrm{p}}=4.95 \pm 0.11 \mathrm{eV}$ and $\Phi_{\mathrm{n}}=4.42 \pm 0.07 \mathrm{eV}$, respectively, and, hence, the performed soft gating increases the work function by $\Delta \Phi_{\mathrm{p}-\mathrm{n}}=530 \mathrm{meV}$. Surprisingly large doping from the residues is extremely important for controlled and reliable device fabrication.

Thus, we show that functional electrical microscopy is a very powerful technique, allowing precise mapping of essential electrical properties on nanoscale. Moreover, the technique is extremely sensitive to the change of the external conditions and influence of atmospheric molecules and processing adsorbates introduced by processing. The research in the area is still in the early development stage and requires a combined, persistent multidisciplinary effort, for example, aiming combination of local electrical and chemical mapping and its correlation with transport properties, to fully realize the potential of graphene in many proposed applications.

\section{Acknowledgements}

This work has been funded by NMS under the IRD Graphene Project (NPL), EMRP under Project 115367 (MetMags) and EU FP7 Project "ConceptGraphene". We are very grateful to Alexander Tzalenchuk, Rositza Yakimova, Jack Patten, Cristina Giusca, Ruth Pearce, Karin Cedergren, Arseniy Lartsev, Steve Spencer and Li Yang for useful discussions and help with the experiments.

\section{Conflict of Interest}

The authors declare no conflict of interest.

\section{References and Notes}

1. Novoselov, K. Nobel lecture: Graphene: Materials in the flatland. Rev. Mod. Phys. 2011, 83, 837-849.

2. Novoselov, K.S.; Fal'ko, V.I.; Colombo, L.; Gellert, P.R.; Schwab, M.G.; Kim, K. A roadmap for graphene. Nature 2012, 490, 192-200.

3. Tzalenchuk, A.; Lara-Avila, S.; Kalaboukhov, A.; Paolillo, S.; Syväjärvi, M.; Yakimova, R.; Kazakova, O.; Janssen, T.J.B.M.; Fal'ko, V.; Kubatkin, S. Towards a quantum resistance standard based on epitaxial graphene. Nat. Nanotechnol. 2010, 5, 186-189.

4. Virojanadara, C.; Syväjarvi, M.; Yakimova, R.; Johansson, L.; Zakharov, A.; Balasubramanian, T. Homogeneous large-area graphene layer growth on $6 \mathrm{H}-\mathrm{SiC}(0001)$. Phys. Rev. B 2008, 78, 245403:1-245403:6.

5. Lin, Y.-M.; Valdes-Garcia, A.; Han, S.-J.; Farmer, D.B.; Meric, I.; Sun, Y.; Wu, Y.; Dimitrakopoulos, C.; Grill, A.; Avouris, P.; Jenkins, K.A. Wafer-scale graphene integrated circuit. Science 2011, 332, 1294-1297.

6. Dimitrakopoulos, C.; Lin, Y.-M.; Grill, A.; Farmer, D.B.; Freitag, M.; Sun, Y.; Han, S.-J.; Chen, Z.; Jenkins, K.A.; Zhu, Y.; Liu, Z.; McArdle, T.J.; Ott, J.A.; Wisnieff, R.; Avouris, P. Wafer-scale epitaxial graphene growth on the $\mathrm{Si}$-face of hexagonal $\mathrm{SiC}(0001)$ for high frequency transistors. J. Vac. Sci. Technol. B Microelectron. Nanometer. Struct. 2010, 28, 985-992. 
7. Moser, J.; Verdaguer, A.; Jiménez, D.; Barreiro, A.; Bachtold, A. The environment of graphene probed by electrostatic force microscopy. Appl. Phys. Lett. 2008, 92, 123507:1-123507:3.

8. Verdaguer, A.; Cardellach, M.; Segura, J.J.; Sacha, G.M.; Moser, J.; Zdrojek, M.; Bachtold, A.; Fraxedas, J. Charging and discharging of graphene in ambient conditions studied with scanning probe microscopy. Appl. Phys. Lett. 2009, 94, 233105:1-233105:3.

9. Burnett, T.L.; Patten, J.; Kazakova, O. Water desorption and re-adsorption on epitaxial graphene studied by SPM. Available online: http://arxiv.org/abs/1204.3323 (accessed on 26 February 2013).

10. Emtsev, K.V.; Bostwick, A.; Horn, K.; Jobst, J.; Kellogg, G.L.; Ley, L.; McChesney, J.L.; Ohta, T.; Reshanov, S.A.; Röhrl, J.; Rotenberg, E.; Schmid, A.K.; Waldmann, D.; Weber, H.B.; Seyller, T. Towards wafer-size graphene layers by atmospheric pressure graphitization of silicon carbide. Nat. Mater. 2009, 8, 203-207.

11. Bolen, M.; Harrison, S.; Biedermann, L.; Capano, M. Graphene formation mechanisms on 4H-SiC(0001). Phys. Rev. B 2009, 80, 115433:1-115433:9.

12. Ferrer, F.J.; Moreau, E.; Vignaud, D.; Deresmes, D.; Godey, S.; Wallart, X. Initial stages of graphitization on $\mathrm{SiC}(000-1)$, as studied by phase atomic force microscopy. J. Appl. Phys. 2011, 109, 054307:1-054307:6.

13. Camara, N.; Tiberj, A.; Jouault, B.; Caboni, A.; Jabakhanji, B.; Mestres, N.; Godignon, P.; Camassel, J. Current status of self-organized epitaxial graphene ribbons on the $\mathrm{C}$ face of $6 \mathrm{H}-\mathrm{SiC}$ substrates. J. Phys. D Appl. Phys. 2010, 43, 374011:1-374011:13.

14. Nonnenmacher, M.; O’Boyle, M.P.; Wickramasinghe, H.K. Kelvin probe force microscopy. Appl. Phys. Lett. 1991, 58, 2921:1-2921:3.

15. Zerweck, U.; Loppacher, C.; Otto, T.; Grafström, S.; Eng, L. Accuracy and resolution limits of Kelvin probe force microscopy. Phys. Rev. B 2005, 71, 125424:1-125424:9.

16. Girard, P. Electrostatic force microscopy: Principles and some applications to semiconductors. Nanotechnology 2001, 12, 485-490.

17. Panchal, V.; Burnett, T.L.; Pearce, R.; Cedergren, K.; Yakimova, R.; Tzalenchuk, A.; Kazakova, O. Surface potential variations in epitaxial graphene devices investigated by Electrostatic Force Spectroscopy. In Proceeding of 12th IEEE Conference on Nanotechnology (IEEE-NANO), Birmingham, UK, 20-23 August 2012.

18. Lu, Y.; Muñoz, M.; Steplecaru, C.; Hao, C.; Bai, M.; Garcia, N.; Schindler, K.; Esquinazi, P. Electrostatic Force Microscopy on oriented graphite surfaces: Coexistence of insulating and conducting behaviors. Phys. Rev. Lett. 2006, 97, 076805:1-076805:4.

19. Datta, S.S.; Strachan, D.R.; Mele, E.J.; Johnson, A.T.C. Surface potentials and layer charge distributions in few-layer graphene films. Nano Lett. 2009, 9, 7-11.

20. Burnett, T.; Yakimova, R.; Kazakova, O. Mapping of local electrical properties in epitaxial graphene using electrostatic force microscopy. Nano Lett. 2011, 11, 2324-2328.

21. Ziegler, D.; Gava, P.; Güttinger, J.; Molitor, F.; Wirtz, L.; Lazzeri, M.; Saitta, A.; Stemmer, A.; Mauri, F.; Stampfer, C. Variations in the work function of doped single- and few-layer graphene assessed by Kelvin probe force microscopy and density functional theory. Phys. Rev. B 2011, 83, 235434:1-235434:7. 
22. Filleter, T.; Emtsev, K.V.; Seyller, T.; Bennewitz, R. Local work function measurements of epitaxial graphene. Appl. Phys. Lett. 2008, 93, 133117:1-133117:3.

23. Filleter, T.; McChesney, J.; Bostwick, A.; Rotenberg, E.; Emtsev, K.; Seyller, T.; Horn, K.; Bennewitz, R. Friction and dissipation in epitaxial graphene films. Phys. Rev. Lett. 2009, 102, 086102:1-086102:4.

24. Curtin, A.E.; Fuhrer, M.S.; Tedesco, J.L.; Myers-Ward, R.L.; Eddy, C.R.; Gaskill, D.K. Kelvin probe microscopy and electronic transport in graphene on $\mathrm{SiC}(0001)$ in the minimum conductivity regime. Appl. Phys. Lett. 2011, 98, 243111:1-243111:3.

25. Xu, K.; Cao, P.; Heath, J.R. Graphene visualizes the first water adlayers on mica at ambient conditions. Science 2010, 329, 1188-1191.

26. Shin, Y.J.; Wang, Y.; Huang, H.; Kalon, G.; Wee, A.T.S.; Shen, Z.; Bhatia, C.S.; Yang, H. Surface-energy engineering of graphene. Langmuir 2010, 26, 3798-3802.

27. Wang, S.; Zhang, Y.; Abidi, N.; Cabrales, L. Wettability and surface free energy of graphene films. Langmuir 2009, 25, 11078-11081.

28. Zhou, H.; Ganesh, P.; Presser, V.; Wander, M.; Fenter, P.; Kent, P.; Jiang, D.; Chialvo, A.; McDonough, J.; Shuford, K.; Gogotsi, Y. Understanding controls on interfacial wetting at epitaxial graphene: Experiment and theory. Phys. Rev. B 2012, 85, 035406:1-035406:11.

29. Rafiee, J.; Mi, X.; Gullapalli, H.; Thomas, A.V.; Yavari, F.; Shi, Y.; Ajayan, P.M.; Koratkar, N.A. Wetting transparency of graphene. Nat. Mater. 2012, 11, 217-222.

30. Ferrari, A.C.; Meyer, J.C.; Scardaci, V.; Casiraghi, C.; Lazzeri, M.; Mauri, F.; Piscanec, S.; Jiang, D.; Novoselov, K.S.; Roth, S.; Geim, A.K. Raman spectrum of graphene and graphene layers. Phys. Rev. Lett. 2006, 97, 187401:1-187401:4.

31. Graf, D.; Molitor, F.; Ensslin, K.; Stampfer, C.; Jungen, A.; Hierold, C.; Wirtz, L. Spatially resolved Raman spectroscopy of single- and few-layer graphene. Nano Lett. 2007, 7, 238-242.

32. Wang, Y.; Ni, Z.H.; Yu, T.; Shen, Z.X.; Wang, H.M.; Wu, Y.H.; Chen, W.; Shen Wee, A.T. Raman studies of monolayer graphene: The Substrate effect. J. Phys. Chem. C 2008, 112, 10637-10640.

33. Lazzeri, M.; Attaccalite, C.; Wirtz, L.; Mauri, F. Impact of the electron-electron correlation on phonon dispersion: Failure of LDA and GGA DFT functionals in graphene and graphite. Phys. Rev. B 2008, 78, 081403:1-081403:4.

34. Casiraghi, C.; Pisana, S.; Novoselov, K.S.; Geim, A.K.; Ferrari, A.C. Raman fingerprint of charged impurities in graphene. Appl. Phys. Lett. 2007, 91, 233108:1-233108:3.

35. Podila, R.; Rao, R.; Tsuchikawa, R.; Ishigami, M.; Rao, A.M. Raman spectroscopy of folded and scrolled graphene. ACS Nano 2012, 6, 5784-5790.

36. Faugeras, C.; Nerrière, A.; Potemski, M.; Mahmood, A.; Dujardin, E.; Berger, C.; de Heer, W.A. Few-layer graphene on SiC, pyrolitic graphite, and graphene: A Raman scattering study. Appl. Phys. Lett. 2008, 92, 011914:1-011914:3.

37. Malard, L.M.; Pimenta, M.A.; Dresselhaus, G.; Dresselhaus, M.S. Raman spectroscopy in graphene. Phys. Rep. 2009, 473, 51-87.

38. Röhrl, J.; Hundhausen, M.; Emtsev, K.V.; Seyller, T.; Graupner, R.; Ley, L. Raman spectra of epitaxial graphene on SiC(0001). Appl. Phys. Lett. 2008, 92, 201918:1-201918:3. 
39. Hibino, H.; Kageshima, H.; Maeda, F.; Nagase, M.; Kobayashi, Y.; Yamaguchi, H. Microscopic thickness determination of thin graphite films formed on $\mathrm{SiC}$ from quantized oscillation in reflectivity of low-energy electrons. Phys. Rev. B 2008, 77, 075413:1-075413:7.

40. Man, K.L.; Altman, M.S. Low energy electron microscopy and photoemission electron microscopy investigation of graphene. J. Phys. Condens. Matter 2012, 24, 314209:1-314209:20.

41. Ohta, T.; El Gabaly, F.; Bostwick, A.; McChesney, J.L.; Emtsev, K.V.; Schmid, A.K.; Seyller, T.; Horn, K.; Rotenberg, E. Morphology of graphene thin film growth on $\mathrm{SiC}(0001)$. New J. Phys. 2008, 10, 023034:1-023034:7.

42. Khokhar, F.S.; Hlawacek, G.; van Gastel, R.; Zandvliet, H.J.W.; Teichert, C.; Poelsema, B. The influence of substrate temperature on growth of para-sexiphenyl thin films on $\operatorname{Ir}\{111\}$ supported graphene studied by LEEM. Surf. Sci. 2012, 606, 475-480.

43. Osaklung, J.; Euaruksakul, C.; Meevasana, W.; Songsiriritthigul, P. Spatial variation of the number of graphene layers formed on the scratched 6H-SiC(0001) surface. Appl. Surf. Sci. 2012, $258,4672-4677$.

44. Jin, L.; Fu, Q.; Zhang, H.; Mu, R.; Zhang, Y.; Tan, D.; Bao, X. Tailoring the growth of graphene on $\mathrm{Ru}(0001)$ via engineering of the substrate surface. J. Phys. Chem. C 2012, 116, 2988-2993.

45. Mathieu, C.; Lalmi, B.; Menteş, T.O.; Pallecchi, E.; Locatelli, A.; Latil, S.; Belkhou, R.; Ouerghi, A. Effect of oxygen adsorption on the local properties of epitaxial graphene on SiC(0001). Phys. Rev. B 2012, 86, 035435:1-035435:5.

46. Matey, J.R.; Blanc, J. Scanning capacitance microscopy. J. Appl. Phys. 1985, 57, 1437:1-1437:8.

47. Isenbart, J.; Born, A.; Wiesendanger, R. The physical principles of scanning capacitance spectroscopy. Appl. Phys. A Mater. Sci. Proc. 2001, 72, S243-S251.

48. Naitou, Y.; Ogiso, H. Capacitive Imaging of Graphene Flakes on $\mathrm{SiO}_{2}$ Substrate. Jpn. J. Appl. Phys. 2011, 50, 066602:1-066602:4.

49. Zhao, S.; Lv, Y.; Yang, X. Layer-dependent nanoscale electrical properties of graphene studied by conductive scanning probe microscopy. Nanoscale Res. Lett. 2011, 6, 498:1-498:6.

50. Giannazzo, F.; Sonde, S.; Nigro, R.; Rimini, E. Mapping the density of scattering centers limiting the electron mean free path in graphene. Nano Lett. 2011, 4612-4618.

51. Lee, J.E.; Ahn, G.; Shim, J.; Lee, Y.S.; Ryu, S. Optical separation of mechanical strain from charge doping in graphene. Nat. Commun. 2012, 3, 1024:1-1024:8.

52. Hass, J.; Millán-Otoya, J.; First, P.; Conrad, E. Interface structure of epitaxial graphene grown on 4H-SiC(0001). Phys. Rev. B 2008, 78, 205424:1-205424:10.

53. Park, J.H.; Mitchel, W.C.; Smith, H.E.; Grazulis, L.; Eyink, K.G. Studies of interfacial layers between 4H-SiC (0001) and graphene. Carbon 2010, 48, 1670-1673.

54. Burnett, T.L.; Yakimova, R.; Kazakova, O. Identification of epitaxial graphene domains and adsorbed species in ambient conditions using quantified topography measurements. J. Appl. Phys. 2012, 112, 054308:1-054308:7.

55. Nemes-Incze, P.; Osváth, Z.; Kamarás, K.; Biró, L.P. Anomalies in thickness measurements of graphene and few layer graphite crystals by tapping mode atomic force microscopy. Carbon 2008, 46, 1435-1442. 
56. Lauffer, P.; Emtsev, K.V.; Graupner, R.; Seyller, T.; Ley, L. Atomic and electronic structure of few-layer graphene on $\mathrm{SiC}(0001)$ studied with scanning tunneling microscopy and spectroscopy. Phys. Rev. B 2008, 77, 155426:1-155426:10.

57. Eriksson, J.; Pearce, R.; Iakimov, T.; Virojanadara, C.; Gogova, D.; Andersson, M.; Syväjärvi, M.; Lloyd Spetz, A.; Yakimova, R. The influence of substrate morphology on thickness uniformity and unintentional doping of epitaxial graphene on SiC. Appl. Phys. Lett. 2012, 100, 241607:1-241607:5.

58. Dimitrakopoulos, C.; Grill, A.; McArdle, T.J.; Liu, Z.; Wisnieff, R.; Antoniadis, D.A. Effect of $\mathrm{SiC}$ wafer miscut angle on the morphology and Hall mobility of epitaxially grown graphene. Appl. Phys. Lett. 2011, 98, 222105:1-222105:3.

59. Low, T.; Perebeinos, V.; Tersoff, J.; Avouris, P. Deformation and scattering in graphene over substrate steps. Phys. Rev. Lett. 2012, 108, 096601:1-096601:4.

60. Robinson, J.; Weng, X.; Trumbull, K.; Cavalero, R.; Wetherington, M.; Frantz, E.; Labella, M.; Hughes, Z.; Fanton, M.; Snyder, D. Nucleation of epitaxial graphene on SiC(0001). ACS Nano 2010, 4, 153-158.

61. Vecchio, C.; Sonde, S.; Bongiorno, C.; Rambach, M.; Yakimova, R.; Raineri, V.; Giannazzo, F. Nanoscale structural characterization of epitaxial graphene grown on off-axis $4 \mathrm{H}-\mathrm{SiC}(0001)$. Nanoscale Res. Lett. 2011, 6, 269:1-269:7.

62. Zhu, W.; Low, T.; Perebeinos, V.; Bol, A.A.; Zhu, Y.; Yan, H.; Tersoff, J.; Avouris, P. Structure and electronic transport in graphene wrinkles. Nano Lett. 2012, 12, 3431-3436.

63. Schmidt, D.; Ohta, T.; Beechem, T. Strain and charge carrier coupling in epitaxial graphene. Phys. Rev. B 2011, 84, 235422:1-235422:8.

64. Haigh, S.J.; Gholinia, A.; Jalil, R.; Romani, S.; Britnell, L.; Elias, D.C.; Novoselov, K.S.; Ponomarenko, L.A.; Geim, A.K.; Gorbachev, R. Cross-sectional imaging of individual layers and buried interfaces of graphene-based heterostructures and superlattices. Nat. Mater. 2012, 11, 764-767.

65. Koutsos, V.; Haschke, H.; Miles, M.J.; Madani, F. Pulling single chains out of a collapsed polymer monolayer in bad-solvent conditions. MRS Proc. 2011, 734, 49-53.

66. Madani-Grasset, F.; Pham, N.T.; Glynos, E.; Koutsos, V. Imaging thin and ultrathin organic films by scanning white light interferometry. Mater. Sci. Eng. B 2008, 152, 125-131.

67. Yavari, F.; Kritzinger, C.; Gaire, C.; Song, L.; Gulapalli, H.; Borca-Tasciuc, T.; Ajayan, P.M.; Koratkar, N. Tunable bandgap in graphene by the controlled adsorption of water molecules. Small 2010, 6, 2535-2538.

68. Kalon, G.; Shin, Y.J.; Yang, H. Tunable metal-insulator transitions in bilayer graphene by thermal annealing. Appl. Phys. Lett. 2011, 98, 233108:1-233108:3.

69. Yang, Y.; Brenner, K.; Murali, R. The influence of atmosphere on electrical transport in graphene. Carbon 2012, 50, 1727-1733.

70. Riedl, C.; Coletti, C.; Starke, U. Structural and electronic properties of epitaxial graphene on SiC(0001): A review of growth, characterization, transfer doping and hydrogen intercalation. $J$. Phys. D Appl. Phys. 2010, 43, 374009:1-374009:17. 
71. Chung, M.G.; Kim, D.H.; Lee, H.M.; Kim, T.; Choi, J.H.; Seo, D.K.; Yoo, J.-B.; Hong, S.-H.; Kang, T.J.; Kim, Y.H. Highly sensitive $\mathrm{NO}_{2}$ gas sensor based on ozone treated graphene. Sens. Actuators B Chem. 2012, 166-167, 172-176.

72. Yoon, H.J.; Jun, D.H.; Yang, J.H.; Zhou, Z.; Yang, S.S.; Cheng, M.M.-C. Carbon dioxide gas sensor using a graphene sheet. Sens. Actuators B Chem. 2011, 157, 310-313.

73. Schedin, F.; Geim, A.K.; Morozov, S.V.; Hill, E.W.; Blake, P.; Katsnelson, M.I.; Novoselov, K.S. Detection of individual gas molecules adsorbed on graphene. Nat. Mater. 2007, 6, 652-655.

74. Wehling, T.O.; Novoselov, K.S.; Morozov, S.V.; Vdovin, E.E.; Katsnelson, M.I.; Geim, A.K.; Lichtenstein, A.I. Molecular doping of graphene. Nano Lett. 2008, 8, 173-177.

75. Zhou, S.; Siegel, D.; Fedorov, A.; Lanzara, A. Metal to insulator transition in epitaxial graphene induced by molecular doping. Phys. Rev. Lett. 2008, 101, 086402:1-086402:4.

76. Sojoudi, H.; Baltazar, J.; Henderson, C.; Graham, S. Impact of post-growth thermal annealing and environmental exposure on the unintentional doping of CVD graphene films. J. Vac. Sci. Technol. B Microelectron. Nanometer. Struct. 2012, 30, 041213:1-041213:6.

77. Llobet, E. Gas sensors using carbon nanomaterials: A review. Sens. Actuators B Chem. 2013, in press.

78. Verdaguer, A.; Sacha, G.M.; Bluhm, H.; Salmeron, M. Molecular structure of water at interfaces: Wetting at the nanometer scale. Chem. Rev. 2006, 106, 1478-510.

79. Kazakova, O.; Burnett, T.L.; Patten, J.; Yang, L.; Yakimova, R. Epitaxial graphene on $\mathrm{SiC}(000-1)$ : Electrical functional microscopy studies and effect of atmosphere. Nanotechnology 2012, submitted for publication.

80. Cao, P.; Xu, K.; Varghese, J.O.; Heath, J.R. The microscopic structure of adsorbed water on hydrophobic surfaces under ambient conditions. Nano Lett. 2011, 11, 5581-5586.

81. Kimmel, G.A.; Matthiesen, J.; Baer, M.; Mundy, C.J.; Petrik, N.G.; Smith, R.S.; Dohnálek, Z.; Kay, B.D. No confinement needed: Observation of a metastable hydrophobic wetting two-layer ice on graphene. J. Am. Chem. Soc. 2009, 131, 12838-12844.

82. Yang, D.-S.; Zewail, A.H. Ordered water structure at hydrophobic graphite interfaces observed by 4D, ultrafast electron crystallography. Proc. Natl. Acad. Sci. USA 2009, 106, 4122-4126.

83. Hibino, H.; Tanabe, S.; Mizuno, S.; Kageshima, H. Growth and electronic transport properties of epitaxial graphene on SiC. J. Phys. D Appl. Phys. 2012, 45, 154008:1-154008:12.

84. Srivastava, N.; He, G.; Mende, P.C.; Feenstra, R.M.; Sun, Y. Graphene formed on SiC under various environments: Comparison of Si-face and C-face. J. Phys. D Appl. Phys. 2012, 45, 154001:1-154001:12.

85. Sun, D.; Divin, C.; Berger, C.; de Heer, W.A.; First, P.N.; Norris, T.B. Spectroscopic measurement of interlayer screening in multilayer epitaxial graphene. Phys. Rev. Lett. 2010, 104, 136802:1-036802:4.

86. Johansson, L.; Watcharinyanon, S.; Zakharov, A.; Iakimov, T.; Yakimova, R.; Virojanadara, C. Stacking of adjacent graphene layers grown on C-face SiC. Phys. Rev. B 2011, 84, 125405:1-125405:8. 
87. Nomani, M.W.K.; Shields, V.; Tompa, G.; Sbrockey, N.; Spencer, M.G.; Webb, R.A.; Koley, G. Correlated conductivity and work function changes in epitaxial graphene. Appl. Phys. Lett. 2012, 100, 092113:1-092113:4.

88. Lin, Y.-M.; Dimitrakopoulos, C.; Farmer, D.B.; Han, S.-J.; Wu, Y.; Zhu, W.; Gaskill, D.K.; Tedesco, J.L.; Myers-Ward, R.L.; Eddy, C.R.; Grill, A.; Avouris, P. Multicarrier transport in epitaxial multilayer graphene. Appl. Phys. Lett. 2010, 97, 112107:1-112107:3.

89. Ryu, S.; Liu, L.; Berciaud, S.; Yu, Y.-J.; Liu, H.; Kim, P.; Flynn, G.W.; Brus, L.E. Atmospheric oxygen binding and hole doping in deformed graphene on a $\mathrm{SiO}_{2}$ substrate. Nano Lett. 2010 , 4944-4951.

90. Leenaerts, O.; Partoens, B.; Peeters, F. Adsorption of $\mathrm{H}_{2} \mathrm{O}, \mathrm{NH}_{3}, \mathrm{CO}, \mathrm{NO}_{2}$, and $\mathrm{NO}$ on graphene: A first-principles study. Phys. Rev. B 2008, 77, 125416:1-125416:6.

91. Zhao, J.; Xiao, B.; Ding, Y. Theoretical prediction of the $\mathrm{N}-\mathrm{H}$ and $\mathrm{O}-\mathrm{H}$ bonds cleavage catalyzed by the single-walled silicon carbide nanotube. J. Phys. Chem. C 2009, 113, 16736-16740.

92. Li, H.; Zeng, X.C. Wetting and interfacial properties of water nanodroplets in contact with graphene and monolayer boron-nitride sheets. ACS Nano 2012, 6, 2401-2409.

93. Gordillo, M.C.; Martí, J. Effect of surface roughness on the static and dynamic properties of water adsorbed on graphene. J. Phys. Chem. B 2010, 114, 4583-4589.

94. Rosso, M.; Arafat, A.; Schroën, K.; Giesbers, M.; Roper, C.S.; Maboudian, R.; Zuilhof, H. Covalent attachment of organic monolayers to silicon carbide surfaces. Langmuir 2008, 24, 4007-4012.

95. Zhuang, H.; Song, B.; Srikanth, V.V.S.S.; Jiang, X.; Schönherr, H. Controlled wettability of diamond $/ \beta-S i C$ composite thin films for biosensoric applications. J. Phys. Chem. C 2010, 114, 20207-20212.

96. Panchal, V.; Cox, D.; Yakimova, R.; Kazakova, O. Epitaxial graphene sensors for detection of small magnetic moments. IEEE Trans. Mag. 2013, 49, 97-100.

97. Panchal, V.; Cedergren, K.; Yakimova, R.; Tzalenchuk, A.; Kubatkin, S.; Kazakova, O. Small epitaxial graphene devices for magnetosensing applications. J. Appl. Phys. 2012, 111, 07E509:1-07E509:3.

98. Goossens, A.M.; Calado, V.E.; Barreiro, A.; Watanabe, K.; Taniguchi, T.; Vandersypen, L.M.K. Mechanical cleaning of graphene. Appl. Phys. Lett. 2012, 100, 073110:1-073110:3.

99. Moser, J.; Barreiro, A.; Bachtold, A. Current-induced cleaning of graphene. Appl. Phys. Lett. 2007, 91, 163513:1-163513:3.

100. Bryan, S.E.; Yang, Y.; Murali, R. Conductance of epitaxial graphene nanoribbons: Influence of size effects and substrate morphology. J. Phys. Chem. C 2011, 115, 10230-10235.

101. Lara-Avila, S.; Moth-Poulsen, K.; Yakimova, R.; Bjørnholm, T.; Fal'ko, V.; Tzalenchuk, A.; Kubatkin, S. Non-volatile photochemical gating of an epitaxial graphene/polymer heterostructure. Adv. Mater. 2011, 23, 878-882.

102. Kopylov, S.; Tzalenchuk, A.; Kubatkin, S.; Fal'ko, V.I. Charge transfer between epitaxial graphene and silicon carbide. Appl. Phys. Lett. 2010, 97, 112109:1-112109:3. 
103. Sonde, S.; Giannazzo, F.; Raineri, V.; Yakimova, R.; Huntzinger, J.-R.; Tiberj, A.; Camassel, J. Electrical properties of the graphene/4H-SiC (0001) interface probed by scanning current spectroscopy. Phys. Rev. B 2009, 80, 241406:1-241406:4.

104. Okudaira, K.K.; Morikawa, E.; Hasegawa, S.; Sprunger, P.T.; Saile, V.; Seki, K.; Harada, Y.; Ueno, N. Radiation damage of poly(methylmethacrylate) thin films analyzed by UPS. J. Electron SpectrosC. Relat. Phenom. 1998, 88-91, 913-917.

105. Ikeura-Sekiguchi, H.; Sekiguchi, T.; Koike, M. Characterization and degradation of ZEP520 resist film by TOF-PSID and NEXAFS. J. Electron Spectrosc. Relat. Phenom. 2005, 144-147, 453-455.

106. Yu, Y.-J.; Zhao, Y.; Ryu, S.; Brus, L.E.; Kim, K.S.; Kim, P. Tuning the graphene work function by electric field effect. Nano Lett. 2009, 9, 3430-3434.

107. Pearce, R. On the differing sensitivity to chemical gating of single and double layer epitaxial graphene explored using Scanning Kelvin Probe Microscopy. ACS Nano 2012, submitted for publication.

108. Bussmann, B.K.; Ochedowski, O.; Schleberger, M. Doping of graphene exfoliated on $\mathrm{SrTiO}_{3}$. Nanotechnology 2011, 22, 265703:1-265703:5.

109. Bruker Cooperation Home Page. Available online: http://www.bruker.com/ (accessed on 27 February 2013).

110. Bruker AFM Probe. Available online: http://www.brukerafmprobes.com/ (accessed on 27 February 2013).

111. Oliver, R.A. Advances in AFM for the electrical characterization of semiconductors. Rep. Prog. Phys. 2008, 71, 076501:1-076501:37.

112. Hao, G.L.; Qi, X.; Li, J.; Yang, L.W.; Yin, J.J.; Lu, F.; Zhong, J.X. Surface potentials of few-layer graphene films in high vacuum and ambient conditions. Solid State Commun. 2011, $151,818-821$.

113. Takagi, A.; Yamada, F.; Matsumoto, T.; Kawai, T. Electrostatic force spectroscopy on insulating surfaces: the effect of capacitive interaction. Nanotechnology 2009, 20, 365501:1-0365501:7.

114. Melitz, W.; Shen, J.; Kummel, A.C.; Lee, S. Kelvin probe force microscopy and its application. Surf. Sci. Rep. 2011, 66, 1-27.

115. Glatzel, T.; Sadewasser, S.; Lux-Steiner, M.C. Amplitude or frequency modulation-detection in Kelvin probe force microscopy. Appl. Surf. Sci. 2003, 210, 84-89.

116. Ziegler, D.; Stemmer, A. Force gradient sensitive detection in lift-mode Kelvin probe force microscopy. Nanotechnology 2011, 22, 075501:1-075501:9.

117. Krok, F.; Sajewicz, K.; Konior, J.; Goryl, M.; Piatkowski, P.; Szymonski, M. Lateral resolution and potential sensitivity in Kelvin probe force microscopy: Towards understanding of the sub-nanometer resolution. Phys. Rev. B 2008, 77, 235427:1-235427:9.

118. Yakimova, R.; Virojanadara, C.; Gogova, D.; Syväjärvi, M.; Siche, D.; Larsson, K.; Johansson, L.I. Analysis of the Formation Conditions for Large Area Epitaxial Graphene on SiC Substrates. Mater. Sci. Forum 2010, 645-648, 565-568.

119. Yakimova, R.; Iakimova, T.; Syväjärvi, M. Process for growth of graphene 2012. WIPO Patent WO2012036608, International Application No. PCT/SE2011/050328, 23 March 2011. 
120. Yannopoulos, S.N.; Siokou, A.; Nasikas, N.K.; Dracopoulos, V.; Ravani, F.; Papatheodorou, G.N. $\mathrm{CO}_{2}$-laser-induced growth of epitaxial graphene on $6 \mathrm{H}-\mathrm{SiC}(0001)$. Adv. Funct. Mater. 2012, 22, 113-120.

121. Luo, Z.; Cong, C.; Zhang, J.; Xiong, Q.; Yu, T. Direct observation of inner and outer G' band double-resonance Raman scattering in free standing graphene. Appl. Phys. Lett. 2012, 100, 243107:1-243107:4.

122. Tiberj, A.; Camara, N.; Godignon, P.; Camassel, J. Micro-Raman and micro-transmission imaging of epitaxial graphene grown on the $\mathrm{Si}$ and $\mathrm{C}$ faces of $6 \mathrm{H}-\mathrm{SiC}$. Nanoscale Res. Lett. 2011, 6, 478:1-478:9.

123. Lee, D.S.; Riedl, C.; Krauss, B.; von Klitzing, K.; Starke, U.; Smet, J.H. Raman spectra of epitaxial graphene on $\mathrm{SiC}$ and of epitaxial graphene transferred to $\mathrm{SiO}_{2}$. Nano Lett. 2008, 8, 4320-4325.

(C) 2013 by the authors; licensee MDPI, Basel, Switzerland. This article is an open access article distributed under the terms and conditions of the Creative Commons Attribution license (http://creativecommons.org/licenses/by/3.0/). 